\title{
EVOLUCIÓN Y ANÁLISIS BIBLIOMÉTRICO DE LA REVISTA CUADERNOS DE TURISMO (1998-2019)
}

\author{
José-Antonio Corral-Marfil* \\ Universidad de Vic-Universidad Central de Cataluña \\ https://orcid.org/0000-0003-0782-3109 \\ Cayetano Espejo Marín** \\ Universidad de Murcia \\ https://orcid.org/0000-0001-6614-1365
}

In memoriam Don José Luis Andrés Sarasa

\section{RESUMEN}

Los objetivos son analizar los artículos publicados en Cuadernos de Turismo y valorar la evolución de la revista. Se estudian los hitos históricos, la distribución de frecuencias de los artículos y las autorías, el origen geográfico e institucional de las autorías, las temáticas y los territorios investigados, el impacto científico, el origen de las citas recibidas y el destino de las citas emitidas. A pesar de la concentración de autorías de España, la revista ha ganado influencia en Iberoamérica. Las temáticas están globalmente en sintonía con la investigación turística internacional. Entre las causas del creciente impacto de la revista, se encuentra la adaptación a los notables cambios que se han producido durante las dos últimas décadas en el entorno de las publicaciones académicas.

Palabras clave: revista científica; turismo; investigación turística; análisis bibliométrico; análisis de contenido; bases de datos bibliográficas; Scopus; Cuadernos de Turismo; España; Iberoamérica.

\section{Evolution and bibliometric analysis of the journal Cuadernos de Turismo (1998-2019)}

\section{ABSTRACT}

The objectives are to analyse the articles published in Cuadernos de Turismo and to assess the journal's evolution. The historical milestones, the frequency distribution of articles

Fecha de recepción: 28 de julio de 2020

Fecha de aceptación: 5 de octubre de 2020

* Departamento de Economía y Empresa. Facultad de Empresa y Comunicación. Universidad de Vic-Universidad Central de Cataluña. C/ Sagrada Familia, 7. 08500 Vic. BARCELONA (España). E-mail: joseantonio. corral@uvic.cat

** Departamento de Geografía. Universidad de Murcia. Campus de la Merced. 30001 MURCIA (España). E-mail: cespejo@um.es 
and authorships, the geographical and institutional origin of authorships, the subjects and territories researched, the scientific impact, and the main cited and citing publications are studied. Despite the concentration of authorships from Spain, the journal has progressively gained influence in Latin America. The subjects are globally consistent with the international tourism research. Among the causes of the journal's growing impact, there is the adaption to the remarkable changes that have occurred in the environment of the academic publishing during the last two decades.

Keywords: Scientific journal; tourism; tourism research; bibliometric analysis; content analysis; bibliographic databases; Scopus; Cuadernos de Turismo; Spain; Ibero-America.

\section{INTRODUCCIÓN}

El turismo, la investigación del turismo y la investigación de la producción científica sobre turismo han aumentado últimamente. En efecto, en primer lugar, hasta la irrupción de la pandemia producida por el coronavirus SARS-CoV-2, el turismo ha crecido sostenidamente a lo largo del tiempo. En 2018, las llegadas mundiales de turistas internacionales aumentaron un 5\% y alcanzaron los 1.400 millones. El turismo es la tercera mayor categoría de exportaciones del mundo, por delante de la automoción y los productos agroalimentarios. España es el segundo destino del mundo por llegadas de turistas internacionales (Organización Mundial del Turismo, 2019). Aparte de impactos económicos, el turismo también causa notables impactos sociales, culturales y medioambientales, positivos y negativos.

Paralelamente, en segundo lugar, durante los últimos 40 años, el campo del turismo ha emergido como un área dinámica de investigación científica, con un crecimiento espectacular en el número de académicos dedicados a la materia, el lanzamiento de nuevas revistas científicas y la publicación de artículos (Gursoy y Sandstrom, 2016). McKercher y Tung $(2015,2016)$ recopilaron de la base de datos Scopus los artículos publicados en revistas especializadas en turismo, hotelería y eventos entre 1980 y 2015 . Hallaron que el número de artículos pasó de 50, publicados en 1980 en dos revistas, a 2.090, publicados en 2015 en 56 revistas; es decir, que el número anual de artículos se multiplicó por 41,8. Eso sin contar los artículos publicados en revistas no especializadas. Actualmente, existen más de 330 revistas arbitradas de turismo, de las cuales unas 200 se publican en inglés (McKercher y Tung, 2016).

En tercer lugar, también han proliferado en los últimos años los estudios bibliométricos que analizan la producción científica en el campo del turismo. Koseoglu et al. (2016) identificaron 190 artículos basados en análisis bibliométricos, publicados en nueve de las principales revistas de turismo y hotelería, entre 1988 y 2015. Detectaron un aumento significativo en el número de estudios bibliométricos a partir de 2008. La mayoría (157) fueron estudios de revisión, 24 fueron estudios evaluativos y solo nueve fueron estudios relacionales. 
Las técnicas empleadas en los estudios de revisión incluyen revisiones estructuradas de la literatura, revisiones sistemáticas y metaanálisis. Los métodos tradicionales de revisión generan conocimiento analizando datos bibliográficos mediante análisis de frecuencias o estadística básica. Las técnicas evaluativas se emplean para cuantificar y comparar el impacto de las contribuciones científicas de diversos individuos o grupos. Se usan indicadores como el número de artículos publicados o citas recibidas, por autores, revistas, universidades o países. Mediante las técnicas relacionales se exploran relaciones entre campos de investigación y la emergencia de nuevos temas o métodos. Se dividen en cuatro categorías: cocitación, coautoría, coincidencia de palabras y emparejamiento bibliográfico (Benckendorff y Zehrer, 2013; Župič y Čater, 2015; Koseoglu et al., 2016).

En turismo los estudios bibliométricos más frecuentes son los de revisión. Koseoglu et al. (2016) los dividen en seis tipos, según si se centran en la disciplina, temas, metodologías, muestras, autores o revistas, aunque inevitablemente existe cierto solapamiento entre ellos. Los centrados en la disciplina evalúan la investigación en el campo del turismo en su conjunto, poniendo énfasis en una o varias dimensiones. Por ejemplo, Park et al. (2016) exploraron la distribución de frecuencias de las áreas temáticas investigadas en turismo, a través del análisis de los artículos publicados en 31 revistas turísticas, en el periodo 2002-2011.

Los estudios centrados en un tema analizan la evolución de un determinado tópico en el conjunto de la disciplina turística; el turismo comunitario (Álvarez-García et al., 2018), el turismo enológico (Durán-Sánchez et al., 2017), la innovación turística (Durán-Sánchez et al., 2019), el smart tourism (Celdrán-Bernabeu et al., 2018) o la Geografía del turismo (Pinassi y Ercolani, 2015).

Los estudios centrados en metodologías examinan la evolución de los métodos de investigación aplicados o los procedimientos estadísticos usados en la literatura turística. Por ejemplo, Xiao y Smith (2006) examinaron el uso del estudio de caso como estrategia de investigación en 76 artículos publicados entre los años 2000 y 2004 en las revistas Annals of Tourism Research, Journal of Travel Research, Tourism Analysis y Tourism Management.

Los estudios centrados en muestras seleccionadas de artículos se focalizan en determinados sectores de actividad, tipologías de personas o lugares. En sectores, como la restauración (Rodríguez-López et al., 2020), o en tipologías de personas, como los niños (Poria y Timothy, 2014). Diversos estudios focalizados en lugares se centran en países, como Brasil y México (Díaz-Carrión y Vizcaíno-Suárez, 2019) o España (Corral-Marfil, 2014; Corral-Marfil y Cànoves, 2016; Peña et al., 2019). Algunos otros se enfocan en regiones, como Iberoamérica (Albach y Medeiros, 2020; Ramón-Cardona y Sánchez-Fernández, 2019), en comunidades autónomas, como Cataluña (Corral-Marfil et al., 2015), o incluso en ciudades, como Girona (Meneguel et al., 2019).

Los estudios centrados en autores a menudo persiguen identificar los autores líderes en la investigación turística. López-Bonilla et al. identificaron la primera generación de autores con difusión internacional en la investigación turística española (2017), elaboraron un ranking de 79 autores prolíficos líderes en investigación turística adscritos a universidades españolas (2018a) y analizaron los autores y los artículos sobre turismo publicados en revistas internacionales de alto impacto (2018b). 
Los estudios de revisión centrados en revistas examinan la evolución de publicaciones específicas, a menudo en ocasión de un aniversario de la revista. Por ejemplo, Tourism (Tokić y Tokić, 2015), Estudios y Perspectivas en Turismo (Osorio, 2016), Journal of Sustainable Tourism (Mauleón-Méndez et al., 2018), Journal of Travel \& Tourism Marketing (Mulet-Forteza et al., 2018), Asia Pacific Journal of Tourism Research (Güzeller y Çeliker, 2019), Tourism Geographies (Merigó et al., 2019), Tourism Management (Qian et al., 2019) y Anatolia (Mokhtari et al., 2020).

No obstante, no se ha publicado ningún estudio bibliométrico específico de la revista Cuadernos de Turismo. Esta omisión representa una laguna notable en la investigación turística, por ser la primera revista universitaria española sobre turismo. En efecto, la revista fue pionera en varios sentidos. Por una parte, cronológicamente, Cuadernos de Turismo fue la primera revista editada por una universidad española. Cuando surgió en 1998, a pesar de la importancia del sector turístico desde hacía décadas, en España solo existían dos revistas, ambas editadas por agencias gubernamentales: Estudios Turísticos, por la Secretaría de Estado de Turismo, y Papers de Turisme, por la Agència Valenciana del Turisme (Espejo, 2012). Por otra parte, hasta 2018, Cuadernos de Turismo fue la única revista turística de España y de Iberoamérica indexada en Scopus, en la categoría Tourism, Leisure and Hospitality Management del Scimago Journal \& Country Rank (SJR) (Ramón-Cardona, 2017).

Durante 22 años Cuadernos de Turismo ha difundido una parte substancial de la investigación científica sobre turismo en español. El hecho de estar accesible libremente en Internet desde muy pronto, como se explica más adelante, dio a la revista gran visibilidad, y cabe pensar que influencia, en Iberoamérica.

En este sentido, en 2019, según Google Analytics, 84.824 usuarios, ubicados en 133 países, consultaron 298.829 páginas del web de la revista, que está alojado en el portal de revistas científicas de la Universidad de Murcia. El 74,6\% de los usuarios eran de fuera de España: 15.077 de México, 10.382 de Ecuador, 8.695 de Perú, 8.323 de Colombia, 4.643 de Argentina, 2.014 de Chile, 1.300 de Brasil, ... (Espejo y García, 2020).

El objetivo del artículo es analizar cuantitativamente los artículos publicados en Cuadernos de Turismo y valorar cualitativamente la trayectoria de la revista. Específicamente, se pretende responder las siguientes preguntas:

1. ¿Cuáles fueron los principales hitos en la evolución de Cuadernos de Turismo?

2. ¿Cómo fue la distribución de frecuencias de los artículos de la revista y las autorías?

3. ¿Cuál fue la procedencia institucional y geográfica de los artículos?

4. ¿De qué temáticas trataron los artículos?

5. ¿Qué territorios se investigaron y qué escalas de análisis se adoptaron?

6. ¿Cuál ha sido el impacto científico de la revista? ¿Qué artículos recibieron más citas? ¿De qué revistas procedieron las citas?

7. ¿Qué autores y revistas fueron los más citados por los artículos de la revista (citas emitidas)? 


\section{LANZAMIENTO Y EVOLUCIÓN DE CUADERNOS DE TURISMO}

La revista fue creada en 1998 por dos profesores del Departamento de Geografía de la Universidad de Murcia: José Luis Andrés Sarasa y Cayetano Espejo Marín. El lanzamiento de la revista coincidió con la adscripción de la Escuela Oficial de Turismo de Murcia a la Universidad de Murcia, propiciada por los cambios legislativos que reformaron la formación en turismo en España a partir de 1996: el Real Decreto 259/1996, sobre la incorporación a la Universidad de los estudios superiores de turismo, y el posterior Real Decreto 604/1996, por el que se estableció el título universitario oficial de Diplomado en Turismo (Ceballos et al., 2010).

Desde su creación, con el ISSN 1139-7861, se ha editado semestralmente por el Servicio de Publicaciones de la Universidad de Murcia. La revista ha tenido dos directores: desde la fundación hasta 2013, José Luis Andrés Sarasa (Figura 1) y, a partir de 2014, Cayetano Espejo Marín, quien hasta entonces fue secretario de redacción. A partir de 2014 Ramón García Marín asumió las tareas de secretario de redacción. Por otra parte, Cayetano Espejo, además, ha sido secretario de redacción de otras tres revistas: Papeles de Geografía, también del Departamento de Geografía de la Universidad de Murcia, entre 1997 y 2003; Nimbus. Revista de Climatología, Meteorología y Paisaje, de la Universidad de Almería, entre 2008 y 2012; y Boletín de la Asociación de Geógrafos Españoles, entre 2010 y 2017.

En la presentación de la revista se enunció un ámbito amplio: "Se pretende comprender, analizar, investigar con seriedad y rigor la problemática derivada del carácter transversal del turismo, porque es un fenómeno social, económico y difuso, que condiciona y a su vez es condicionado por una pluralidad de sectores y factores (Andrés, 1998, p. 7) (...) "sean bienvenidas todas las colaboraciones desde los más diversos ángulos del pensamiento que puedan aportar luz, imaginación, crítica y nuevos conocimientos a la problemática transversal y transdisciplinar del turismo" (Andrés, 1998, p. 8).

Hasta el número 6, según las normas para la publicación de originales de la revista, los únicos límites del alcance eran el carácter científico y la temática turística de los artículos: "Da cabida a aportaciones científicas relacionadas con el turismo" (Cuadernos de Turismo, 1998, p. 151). Sin embargo, a partir del número 7, aunque el ámbito de la revista se mantuvo amplio, se delimitó de la siguiente forma: "Cuadernos de Turismo es una publicación semestral dirigida a universidades, organismos nacionales e internacionales, profesionales y personas interesadas en el estudio del turismo. Su temática abarca aspectos geográficos, urbanísticos, económicos, ecológicos y formativos relativos a la actividad turística" (Cuadernos de Turismo, 2001).

Con respecto al alcance geográfico, aunque al comienzo predominaron los estudios desde y sobre la Región de Murcia, con el paso del tiempo el origen de los artículos se fue diversificando. En efecto, en la presentación se declaraba: "pretende abrir sus páginas a todo enfoque racional, técnico, serio y documentado de la problemática que ocupa al sector turístico en general, pero de un modo particular al turismo de la Región de Murcia, al que se desea servir sin desmayo porque a él nos debemos por muchas razones" (Andrés, 1998, pp. 7-8). Pero pasados 15 años desde el nacimiento ya se afirmaba que "mientras que en los números iniciales casi la totalidad de autores eran de Murcia, hace varios años 
que la presencia de autores de instituciones murcianas es muy reducida en comparación con la de otras instituciones de otras regiones y países (Espejo, 2012, p. 57).

\section{Figura 1}

FOTOGRAFÍA DE DON JOSÉ LUIS ANDRÉS SARASA, CATEDRÁTICO

DE GEOGRAFÍA HUMANA DE LA UNIVERSIDAD DE MURCIA, EN EL

NÚMERO ESPECIAL DE CUADEROS DE TURISMO $(27,2011)$ QUE SE LE DEDICÓ COMO HOMENAJE CON MOTIVO DE SU JUBILACIÓN

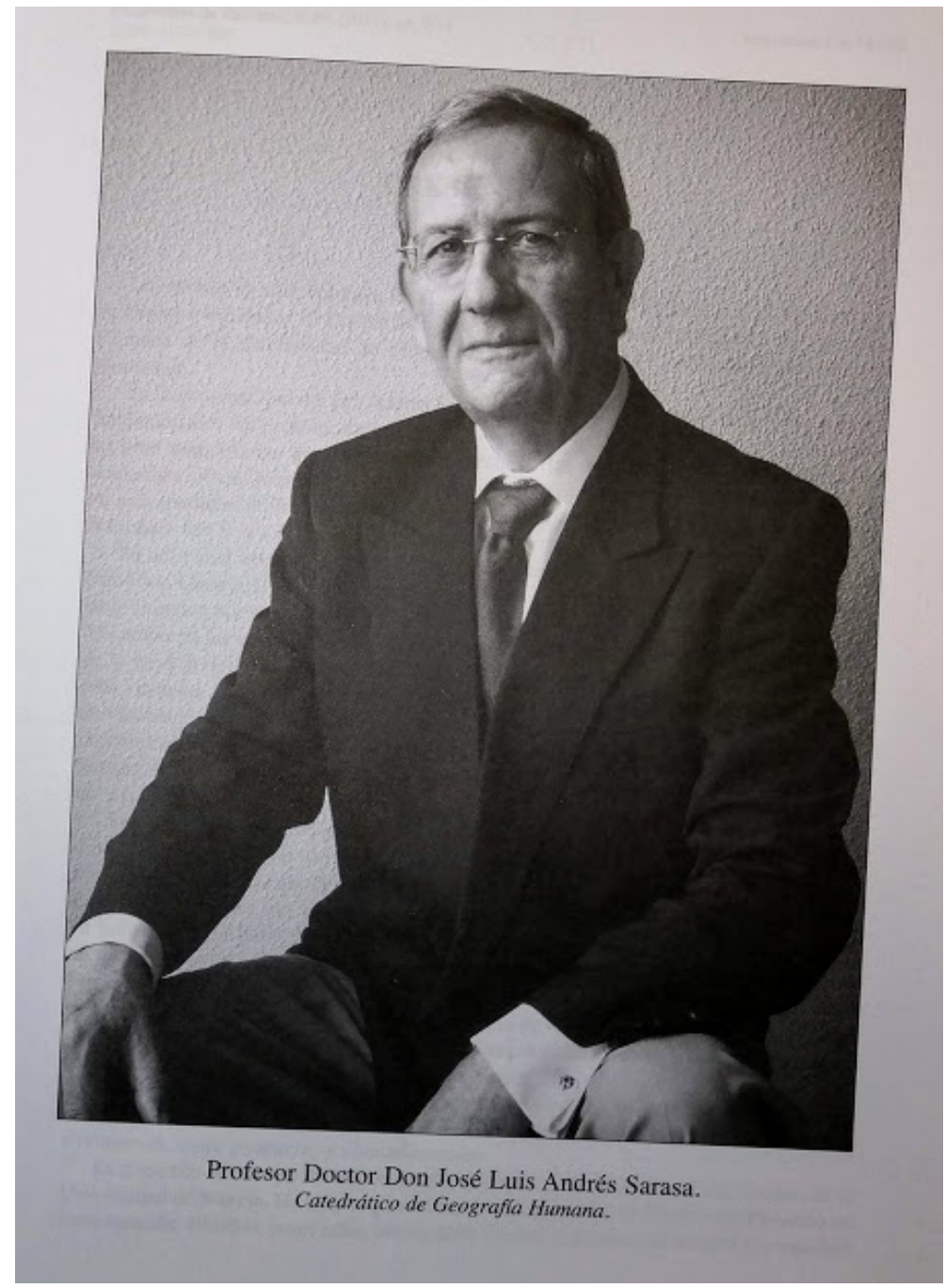

Fuente: Cuadernos de Turismo. 
En cuanto al idioma en que se han publicado los artículos, desde el número 1 hasta el número 22, las normas de publicación solicitaban "el resumen del artículo y las palabras clave, en castellano, y en inglés o francés" (Cuadernos de Turismo, 1998). Sin embargo, no hacían referencia explícita al idioma de los artículos en sí, hecho que permite deducir que debían entregarse en castellano. A partir del número 23, se especificó en las normas que "se publicarán textos escritos en castellano e inglés" (Cuadernos de Turismo, 2009); además, se incluyeron las normas en inglés (Rules for Authors) y un índice con los títulos de los artículos en inglés (Summary). A partir del número 24 se comienza a publicar un resumen amplio en inglés de cada artículo, el cual se exige en la normas a partir del número 26: "Cuando el artículo haya sido aceptado se presentará un resumen amplio del mismo - entre 3 y 5 páginas (máx. 21.000 caracteres con espacios incluidos) y siguiendo la tipología de texto indicada - íntegramente en inglés (o en castellano, para aquellos trabajos presentados originalmente en inglés) que se publicará en la segunda parte de la revista" (Cuadernos de Turismo, 2010).

Pronto se adoptó la filosofía de acceso abierto (open access) y se distribuyeron los artículos en línea, gratuitamente y sin barreras. En 2004 los artículos estuvieron disponibles en el web del Departamento de Geografía de la Universidad de Murcia y en 2005 en el Portal de revistas científicas de la Universidad de Murcia. En 2005 y 2006 se incluyó en dos portales de acceso abierto de difusión de la producción científica hispana; respectivamente, Redalyc -Red de Revistas Científicas de América Latina y el Caribe- y Dialnet, de la Universidad de La Rioja. "También ha sido muy importante el papel de las bases de datos bibliográficos Dialnet y Redalyc, a las que nos incorporamos cuando supimos de su existencia, y que tanto han contribuido a la difusión de los artículos publicados en nuestra revista." (Espejo, 2012, p. 57).

La impresión y difusión en papel se fue reduciendo a lo largo del tiempo hasta eliminarse: "Otra gran labor de difusión se ha llevado a cabo desde los primeros números con el envío de ejemplares a instituciones y profesionales que investigan sobre turismo desde diversos ámbitos" (Espejo, 2012, p. 57). Durante los primeros 14 años (1998-2011) se imprimieron en papel "varios centenares de ejemplares de cada número, de los que se destinaban 120 a las bibliotecas de universidades e instituciones de una veintena de países. Desde la Secretaría de Redacción se enviaban más de 200 ejemplares de cada número publicado a personas vinculadas con la revista, preferentemente por ser evaluadores. Otra parte de las revistas editadas las recibían los autores que publicaban en cada número. Desde la Consejería de Turismo de la Comunidad Autónoma de la Región de Murcia también se remitían a las Concejalías de Turismo de los Ayuntamientos de la Región, y a otros destinos que se consideraba oportuno" (Espejo, 2012, p. 59).

A partir de 2012, se redujo drásticamente la tirada en papel, a raíz del fin de la subvención que la Consejería de Turismo había concedido a la revista desde el principio, a cambio de insertar publicidad de Murcia Turística en la portada. "Esto lleva a plantear una nueva estrategia, dirigida a editar un número de ejemplares en papel que sea el realmente necesario. Teniendo en cuenta su enorme difusión, y sobre todo la facilidad para poder disponer de sus contenidos en Internet, con un reducido número de ejemplares en papel es suficiente para destinarlos a las bases de datos bibliográficos y a los autores que los solicitan" (Espejo, 2012, p. 59). Finalmente, a partir de 2019 la revista dejó de 
publicarse en papel: el número 42 fue el último que se imprimió en papel (Figura 2) y el número 43 se publicó únicamente en formato PDF.

Figura 2

LOS 42 NÚMEROS DE CUADERNOS DE TURISMO IMPRESOS EN PAPEL
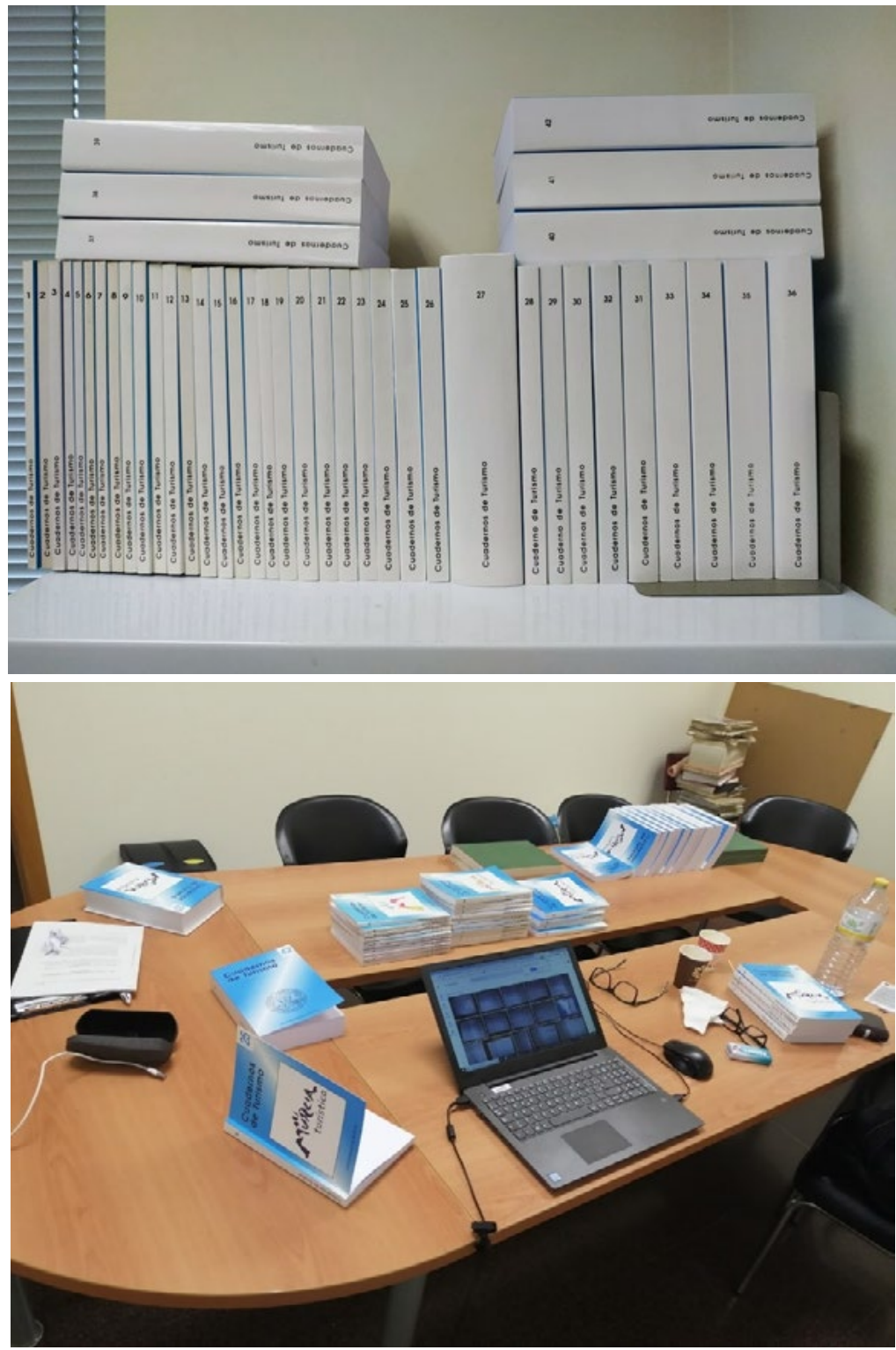

Fuente: Elaboración propia. 


\subsection{Bases de datos, índices y rankings}

La presencia de una revista en bases de datos puede usarse como un indicio de calidad complementario a los indicadores bibliométricos (FECYT, 2018). El Anexo 1 muestra todas las bases de datos e índices que han incluido Cuadernos de Turismo a lo largo del tiempo. En este apartado se comenta la presencia de la revista en bases de datos destacadas; a saber, Scopus, Emerging Sources Citation Index (ESCI), Sello de Calidad y Ranking FECYT $^{1}$, Índice H según Google Scholar Metrics, Matriz de Información para el Análisis de Revistas (MIAR), Clasificación Integrada de Revistas Científicas (CIRC), clasificación Qualis y Clasificación de Revistas Científicas de los ámbitos de las Ciencias Sociales y Humanidades (Carhus Plus+).

En 2013 la revista fue incorporada a Scopus, la base de datos bibliográfica de resúmenes y citas de Elsevier. Fue incluida en tres categorías temáticas: Tourism, Leisure and Hospitality Management (dentro del área temática Business, Management and Accounting), Nature and Landscape Conservation (dentro de Environmental Science) y Geography, Planning and Development (Social Sciences). Países de las revistas de turismo de Scopus

En 2018 hubo 102 revistas indexadas en la categoría temática Tourism, Leisure and Hospitality Management, de las cuales 51 eran británicas, 28 estadounidenses y las 23 restantes de otros países. Hasta 2018, Cuadernos de Turismo fue la única revista turística de España y de Iberoamérica indexada en Scopus (Ramón-Cardona, 2017), y la primera en la categoría Tourism, Leisure and Hospitality Management del Scimago Journal \& Country Rank (SJR). Cuadernos de Turismo ingresó en Scopus en 2013. Las otras dos revistas turísticas españolas indexadas en Scopus ingresaron en 2018: Investigaciones Turísticas, de la Universidad de Alicante, y Enlightening Tourism, de la Universidad de Huelva. A mediados de 2020 ingresa la Revista de Análisis Turístico, de la Asociación Española de Expertos Científicos en Turismo. Por otra parte, ninguna revista turística latinoamericana está indexada en Scopus.

En 2015 Cuadernos de Turismo fue incluida en la base de datos ESCI, que forma parte de la plataforma Web of Science Core Collection, de Clarivate Analytics; en las categorías Social Sciences, General y Hospitality, Leisure, Sport \& Tourism (Web of Science Master Journal List, 2020). ESCI "extiende el universo de publicaciones de Web of Science para incluir publicaciones de alta calidad, revisadas por pares, de importancia regional y en campos científicos emergentes" (Clarivate Analytics, 2017). Las revistas incluidas en esta lista son continuamente evaluadas para su inclusión en Science Citation Index Expanded (SCIE), Social Sciences Citation Index (SSCI) y Arts \& Humanities Citation Index (AHCI). En mayo de 2020, otras tres revistas de turismo españolas estaban incluidas en ESCI: Gran Tour, Investigaciones Turísticas y Pasos. Revista de Turismo y Patrimonio Cultural; sin embargo, ninguna revista española de turismo estaba incluida en los índices SCIE, SSCI o AHCI.

1 En el Ranking de Visibilidad e Impacto de Revistas Científicas Españolas de Humanidades y Ciencias Sociales con Sello de Calidad FECYT, en el año 2019 Cuadernos de Turismo está en el primer cuartil en Economía (posición 3 de 24) y en el primer cuartil en Geografía (posición 3 de 20). https://calidadrevistas.fecyt.es/ ranking. 
En 2019 Fundación Española para la Ciencia y la Tecnología (FECYT) renovó el Sello de Calidad FECYT a Cuadernos de Turismo, en la VI Edición de la Convocatoria de Evaluación de Revistas Científicas Españolas 2018, reconocimiento que la revista había obtenido en 2012. En 2019, además de Cuadernos de Turismo, otras dos revistas de turismo españolas contaban con el citado Sello: Pasos. Revista de Turismo y Patrimonio Cultural e Investigaciones Turísticas (FECYT, 2020). En el conjunto de convocatorias, la FECYT había evaluado 1.035 revistas diferentes y el número de revistas distinguidas con el Sello de Calidad FECYT era de 396. El Sello de Calidad FECYT reconoce las calidades editorial y científica, y está basado en el cumplimiento de 19 indicadores (FECYT, 2018).

La FECYT, además de evaluar la calidad de las revistas españolas mediante el otorgamiento del Sello de Calidad FECYT, elabora un ranking de visibilidad e impacto de revistas científicas españolas de humanidades y ciencias sociales con Sello de Calidad FECYT. La clasificación se basa en indicadores de visibilidad (presencia en bases de datos) y de impacto (citas recibidas). En el ranking de 2018, Cuadernos de Turismo ocupó la quinta posición de las 20 revistas de la categoría temática de geografía, esto es, se ubicó en el primer cuartil, con una puntuación de 51,42 (FECYT, 2020).

El Índice $\mathrm{H}$ de las revistas científicas españolas según Google Scholar Metrics del período 2014-2018 clasificó a Cuadernos de Turismo en el segundo puesto de 30 revistas de la disciplina de Geografía y en el quinto puesto de 81 revistas de la disciplina de Economía y Empresa, ambas disciplinas se encuadran en el campo científico de las ciencias sociales (Delgado y Martín-Martín, 2019). En 2019 el Índice h5 de Cuadernos de Turismo fue 15, lo que significa que como mínimo 15 artículos de la revista fueron citados por lo menos 15 veces cada uno, durante 5 años anteriores (2014-2018). La mediana h5 fue 22, la mitad de los 15 artículos anteriores recibieron 22 o más citas (Google Scholar Metrics).

MIAR es una base de datos de la Universidad de Barcelona que reúne información sobre la presencia de más de 40.000 revistas en bases de datos científicas y repertorios (Universidad de Barcelona, 2020). En base a esa información, y mediante un sistema de puntuación, MIAR calcula el Índice Compuesto de Difusión Secundaria (ICDS) de las revistas, que es un indicador de su visibilidad. Cuanto mayor es el ICDS de una revista, mayor es su presencia en bases de datos de relevancia internacional. La Figura 3 muestra el registro MIAR de Cuadernos de Turismo del año 2019, cuando obtuvo un ICDS de 9.8 .

CIRC de EC3metrics consiste en una valoración sintética de revistas científicas de ciencias sociales y humanas. Se basa en la valoración que reciben las revistas en una serie de bases de datos de referencia y sigue criterios que aplican las agencias de evaluación científica españolas, CNEAI y ANECA, en los procesos de evaluación de profesores. CIRC clasifica las revistas en cinco grupos: A+, A, B, C y D. En 2019 incluyó Cuadernos de Turismo en el grupo B: "Revistas científicas de calidad pero que no alcanzan un alto nivel de internacionalización" (EC3metrics, 2020).

Además, Cuadernos de Turismo, en el sistema Qualis, del Conselho de Aperfeiçoamento de Pessoal do Ensino Superior, del Ministerio de Educación de Brasil, fue clasificada en el nivel B1 en el área de evaluación Administração pública e de empresas, ciências contábeis 
e turismo, en el cuatrienio 2013-2016 (CAPES, 2016). Por último, en Carhus Plus+ 2014, de la Agència de Gestió d'Ajuts Universitaris i de Recerca de la Generalitat de Catalunya, fue clasificada en el grupo D, dentro del ámbito de la Economía (AGAUR, 2014).

\section{Figura 3 \\ REGISTRO MIAR DE CUADERNOS DE TURISMO EN 2019}

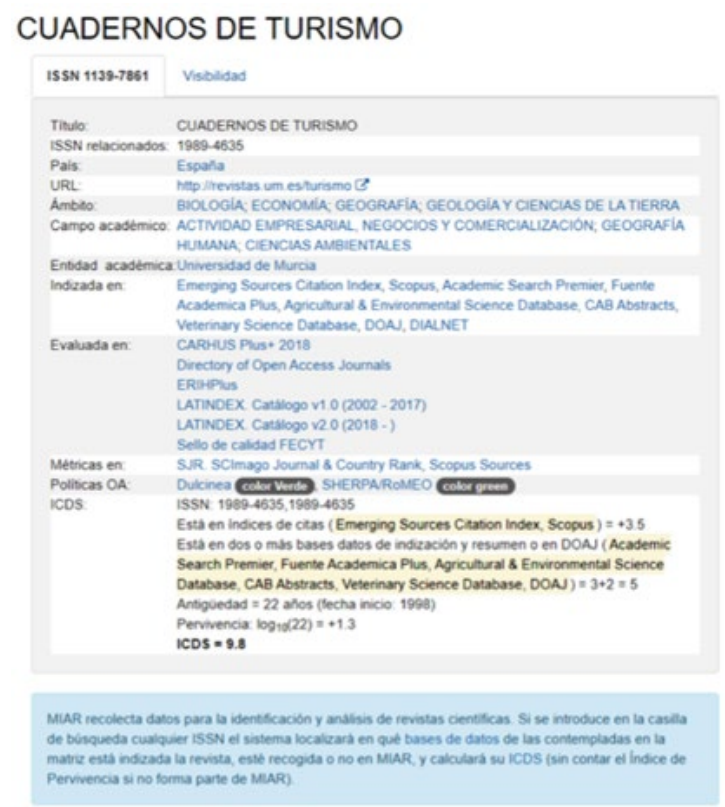

Fuente: http://miar.ub.edu/issn/1139-7861

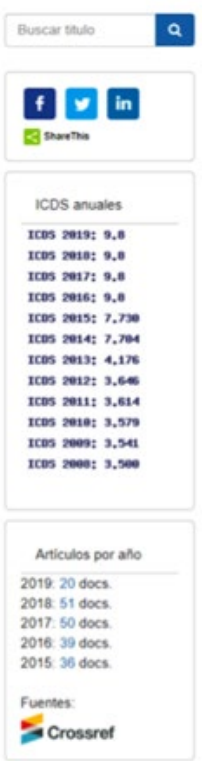

\section{METODOLOGÍA DEL ESTUDIO BIBLIOMÉTRICO}

El estudio consta de cuatro partes: un análisis bibliométrico de las autorías y la procedencia de los artículos publicados semestralmente durante los primeros 22 años de la revista (1998-2019); una clasificación de las palabras clave, para explorar las temáticas tratadas, los territorios investigados y las metodologías seguidas; una revisión de las bases de datos, índices y rankings que incluyen la revista; y un examen del impacto científico de la publicación y de las citas recibidas y emitidas.

Los artículos originales se han descargado en formato PDF del web https://revistas. um.es/turismo/issue/archive. La información sobre las bases de datos bibliográficas se obtuvo de la revista impresa en papel y de los respectivos webs de las bases de datos. En los 44 números editados, se han seleccionado 609 artículos para el análisis; tras excluir 13 reseñas bibliográficas, dos índices, dos presentaciones y una necrología, y se han incluido dos notas de investigación. 
De cada artículo se han codificado los siguientes atributos: número de la revista, año de publicación, número de firmas, nombres de los autores, afiliación de los autores (institución, país y comunidad autónoma) y palabras clave. Los nombres completos de los autores se han normalizado para evitar duplicidades. A los autores con afiliaciones diferentes en artículos diferentes, se les ha asignado la afiliación más reciente. Las afiliaciones se han clasificado entre universidades, empresas e instituciones gubernamentales; y se han registrado al nivel organizativo superior: de las empresas se ha registrado la matriz en lugar de la filial, y de las universidades se ha registrado la universidad en lugar de la facultad, la escuela adscrita o el departamento.

Al contar el número de artículos de autores, instituciones, países y comunidades autónomas, se ha aplicado el método del conteo de casos (instance counting), según el cual un artículo firmado por dos autores otorga "un punto" (autoría, firma) a cada autor (o institución o país o comunidad autónoma), y no medio punto a cada autor (fractional counting), en línea con otros estudios bibliométricos (Severt et al., 2009; Corral-Marfil, 2013).

Las temáticas de estudio, los métodos utilizados y los territorios investigados se han analizado a través de las palabras clave de los artículos. En primer lugar, se han revisado las palabras clave de los artículos para detectar y fusionar términos expresados a través de diversas variaciones lingüísticas, como singular y plural, abreviaturas, variaciones dialectales, expresiones incompletas, etc. En otros, se han fusionado los términos: viajes y viaje, percepciones sociales y percepción social, sistemas de información geográfica y SIG, pequeñas y medianas empresas y pymes, turismo sustentable y turismo sostenible, recreo y recreación, atracción turística y atractivo turístico, ordenación del territorio y ordenación territorial, imagen del destino e imagen de destinos turísticos, Q de Calidad y Q de Calidad Turística, etc.

A continuación, se han clasificado las palabras clave siguiendo el marco de categorización del sistema de investigación turístico de Park et al. (2016), basado en Mill y Morrison (2006), que consta de seis categorías principales -destino, marketing, demanda, viaje, términos genéricos y áreas geográficas- y 61 subcategorías. Para poder clasificar mejor algunas palabras clave, se han añadido las subcategorías destino, demanda y género a las categorías destino, demanda y términos genéricos, respectivamente. Cada palabra clave se ha clasificado en una sola subcategoría.

En la categoría áreas geográficas se han clasificado las palabras clave que aludían a nombres propios de lugar o topónimos. A su vez, los topónimos se han sometido a una doble clasificación. Por una parte, según la ubicación de los lugares que denominan: los de fuera de España, por continentes y países, y los de España, por comunidades autónomas y provincias. Por la otra, según el nivel territorial administrativo que denominan: municipal, supramunicipal, provincial, supraprovincial, autonómico, estatal o supraestatal.

A continuación, una vez clasificados los atributos de los artículos, se han analizado mediante un estudio estadístico descriptivo univariante con el paquete estadístico SPSS. Se han confeccionado representaciones gráficas mediante los programas Excel y Power Point de Microsoft, nubes de palabras clave mediante la aplicación en línea www. 
wordclouds.com y mapas con la herramienta en línea www.instamaps.cat del Institut Cartogràfic i Geològic de Catalunya.

La información sobre el impacto científico general de la revista se ha obtenido del ranking SCImago Journal \& Country Rank (www.scimagojr.com) y ha estado referida al periodo 2014-2018. Se han consultado el indicador SJR, el cuartil y el índice H. Por otra parte, para analizar específicamente las citas recibidas y emitidas por la revista, se ha consultado la base de datos Scopus (www.scopus.com). Se han localizado los artículos de Cuadernos de Turismo indexados en Scopus mediante la búsqueda "Cuadernos de Turismo" en el campo Sourcetitle. La información sobre los artículos más citados y el origen (revistas) de las citas recibidas se ha obtenido por medio de la funcionalidad View cited by, y ha estado referida al periodo comprendido entre el año de entrada de la revista en Scopus (2013) y la fecha de recolección de la información (1 de mayo de 2020). La información sobre las revistas y los autores más citados en los artículos de Cuadernos de Turismo (citas emitidas) se ha obtenido mediante la funcionalidad View references, y comprende el periodo 2013-2019.

\section{RESULTADOS}

\subsection{Autoría y procedencia de los artículos}

\subsubsection{Autores y autoras}

En los 44 números de la revista se han publicado un total de 609 artículos, esto es, una media de13,8 artículos por ejemplar. Ha habido tres números especiales: el 14 (2004), dedicado al turismo de interior en la Región de Murcia; el 18 (2006), dedicado al turismo de peregrinación en España; y el 27, dedicado al Profesor Don José Luis Andrés Sarasa (Espejo, 2011). La Figura 4 muestra la evolución del número de artículos por ejemplar. Aproximadamente, en los primeros 26 números se han publicado alrededor de 10 artículos por ejemplar. El número 27 cuenta con 50 artículos, debido a que se trata del número especial dedicado al Homenaje al Catedrático de Geografía Humana de la Universidad de Murcia y fundador de Cuadernos de Turismo, Doctor Don José Luis Andrés Sarasa, con motivo de su jubilación. A partir del número 28, el número de artículos por ejemplar aumentó gradualmente, hasta estabilizarse en los 20 artículos en los últimos números.

Los 609 artículos cuentan con 1.208 autorías o firmas. Casi el 40\% de los artículos han sido firmados por un solo autor, y más del $70 \%$ por uno o dos autores (Tabla 1). En promedio cada artículo ha tenido 1,98 firmas. El número medio de firmas por artículo ha sido de 1,67 en los primeros 11 años (1998-2008), mientras que asciende a 2,14 en los siguientes 11 años (2009-2019), lo que parece indicar que, a pesar del bajo grado de colaboración global, se ha producido un cierto aumento de la colaboración a lo largo del tiempo. 


\section{Figura 4 \\ EVOLUCIÓN DEL NÚMERO DE ARTÍCULOS POR EJEMPLAR DE LA REVISTA}

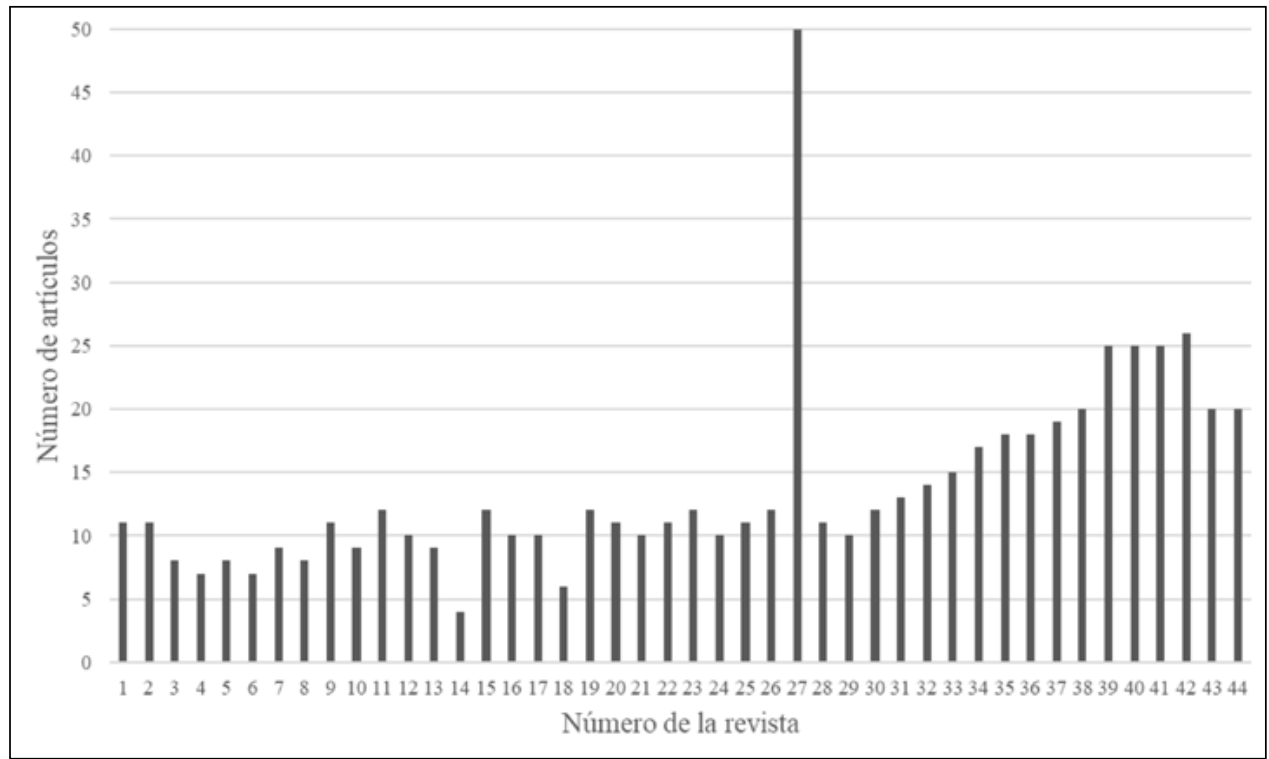

Fuente: Elaboración propia.

Tabla 1

NÚMERO DE FIRMAS POR ARTÍCULO

\begin{tabular}{cccc}
\hline Autorías/artículo & Artículos & \% Artículos & \% Acum. artículos \\
\hline 1 & 240 & 39,4 & 39,4 \\
\hline 2 & 194 & 31,9 & 71,3 \\
\hline 3 & 129 & 21,2 & 92,4 \\
\hline 4 & 39 & 6,4 & 98,9 \\
\hline 5 & 5 & 0,8 & 99,7 \\
\hline 6 & 2 & 0,3 & 100,0 \\
\hline Total & 609 & 100 & \\
\hline
\end{tabular}

Fuente: Elaboración propia.

Han publicado un total 863 autores diferentes, de los cuales el 78,7\% lo han hecho con un solo artículo en la revista, el 11,9\% han publicado dos artículos, el 5,2\% tres artículos, el 1,9\% cuatro artículos y el 2,3\% entre cinco y diez artículos. Entre éstos se encuentran los 20 autores que más artículos han publicado en Cuadernos de Turismo (Tabla 2). 


\section{Tabla 2 \\ AUTORAS Y AUTORES CON MÁS DE CUATRO FIRMAS EN CUADERNOS DE TURISMO}

\begin{tabular}{llc}
\hline Autor o autora & Institución & $\begin{array}{c}\text { Núm. } \\
\text { de } \\
\text { firmas }\end{array}$ \\
\hline Andrés Sarasa, José Luis & Universidad de Murcia & 10 \\
\hline Sánchez Crispín, Álvaro & Universidad Nacional Autónoma de México & 9 \\
\hline Millán Escriche, Mercedes & Universidad de Murcia & 9 \\
\hline Cànoves Valiente, Gemma & Universitat Autònoma de Barcelona & 9 \\
\hline Ponce Sánchez, María Dolores & Universidad de Murcia & 8 \\
\hline Cebrián Abellán, Aurelio & Universidad de Murcia & 7 \\
\hline Propín Frejomil, Enrique & Universidad Nacional Autónoma de México & 6 \\
\hline Espejo Marín, Cayetano & Universidad de Murcia & 6 \\
\hline Such Devesa, María Jesús & Universidad de Alcalá & 5 \\
\hline Serrano Martínez, José María & Universidad de Murcia & 5 \\
\hline Royo Vela, Marcelo & Universitat de València & 5 \\
\hline Pulido Fernández, Juan Ignacio & Universidad de Jaén & 5 \\
\hline Ortega Martínez, Enrique & Universidad Complutense de Madrid & 5 \\
\hline López-Guzmán Guzmán, Tomás Jesús & Universidad de Córdoba & 5 \\
\hline López Olivares, Diego & Universitat Jaume I & 5 \\
\hline Hortelano Mínguez, Luis Alfonso & Universidad de Salamanca & 5 \\
\hline García Hernández, María & Universidad Complutense de Madrid & 5 \\
\hline Feo Parrondo, Francisco & Universidad Autónoma de Madrid & 5 \\
\hline Belmonte Serrato, Francisco & Universidad de Murcia & 5 \\
\hline Araque Jiménez, Eduardo & Universidad de Jaén & 5 \\
\hline & & 5 \\
\hline
\end{tabular}

Fuente: Elaboración propia.

\subsubsection{Procedencia institucional y geográfica de los artículos}

De las 1.208 autorías, la inmensa mayoría $(97,1 \%)$ son autores afiliados a universidades; el resto correspondieron a autores pertenecientes a instituciones gubernamentales $(1,7 \%)$ o empresas $(1,2 \%)$. En total se han contabilizado autores de 184 instituciones, de las cuales 15 concentran la mitad de las autorías (49,9\%) (Tabla 3). Las universidades de Murcia, Alicante, Sevilla y Castilla-La Mancha son las que más autorías han aportado, casi una cuarta parte del total $(24,0 \%)$. 


\section{Tabla 3 \\ INSTITUCIONES CON MÁS DE 14 AUTORÍAS}

\begin{tabular}{|c|c|c|c|}
\hline Afiliación & Autorías & \% Autorías & \% Acum. autorías \\
\hline Universidad de Murcia & 129 & 10,7 & 10,7 \\
\hline Universidad de Alicante & 66 & 5,5 & 16,1 \\
\hline Universidad de Sevilla & 52 & 4,3 & 20,4 \\
\hline Universidad de Castilla-La Mancha & 43 & 3,6 & 24,0 \\
\hline Universidad de La Laguna & 34 & 2,8 & 26,8 \\
\hline Universidad de Málaga & 33 & 2,7 & 29,6 \\
\hline Universidad Autónoma de Madrid & 30 & 2,5 & 32,0 \\
\hline Universidad de Las Palmas de Gran Canaria & 30 & 2,5 & 34,5 \\
\hline Universidad Rey Juan Carlos & 29 & 2,4 & 36,9 \\
\hline Universidad Complutense de Madrid & 28 & 2,3 & 39,2 \\
\hline Universidad Nacional Autónoma de México & 28 & 2,3 & 41,6 \\
\hline Universitat de València & 27 & 2,2 & 43,8 \\
\hline Universidade de Vigo & 25 & 2,1 & 45,9 \\
\hline Universitat Autònoma de Barcelona & 25 & 2,1 & 47,9 \\
\hline Universitat de Lleida & 24 & 2,0 & 49,9 \\
\hline Universidad de Córdoba & 23 & 1,9 & 51,8 \\
\hline Universitat de les Illes Balears & 22 & 1,8 & 53,6 \\
\hline Universidad de Cádiz & 20 & 1,7 & 55,3 \\
\hline Universidade de Santiago de Compostela & 20 & 1,7 & 57,0 \\
\hline Universitat de Girona & 18 & 1,5 & 58,4 \\
\hline Universitat Jaume I & 18 & 1,5 & 59,9 \\
\hline Universitat Rovira i Virgili & 18 & 1,5 & 61,4 \\
\hline Universidad de Jaén & 17 & 1,4 & 62,8 \\
\hline Universidad de Alcalá & 16 & 1,3 & 64,2 \\
\hline Universidad de Cantabria & 16 & 1,3 & 65,5 \\
\hline Universidad de Extremadura & 15 & 1,2 & 66,7 \\
\hline Universidad de Salamanca & 15 & 1,2 & 68,0 \\
\hline Universidad Politécnica de Cartagena & 15 & 1,2 & 69,2 \\
\hline $\begin{array}{l}\text { Otras } 156 \text { instituciones con menos de } 15 \\
\text { autorías }\end{array}$ & 372 & 30,8 & 100,0 \\
\hline Total & 1.208 & 100,0 & \\
\hline
\end{tabular}

Fuente: Elaboración propia. 
Los autores de los artículos están adscritos a instituciones de 25 países. El principal ha sido España, que ha aportado 981 de las 1.208 autorías $(81,2 \%)$; el segundo México, con 83 autorías $(6.9 \%)$. Sólo otros cuatro países cuentan con más de 10 autorías: Argentina $(27 ; 2,2 \%)$, Brasil $(20 ; 1,7 \%)$, Ecuador $(11 ; 0,9 \%)$ y Francia $(11 ; 0,9 \%)$. El resto de los países han aportado menos de 10 autorías: Cuba, Portugal, Colombia, Venezuela, Chile, Italia, Marruecos, Uruguay, Reino Unido, Canadá, Bulgaria, Estados Unidos, Eslovenia, Irlanda, Japón, Nicaragua, Puerto Rico, República Dominicana y Tailandia.

En el caso de España, hay autorías procedentes de las 17 comunidades autónomas. Las que más contribuyen son las situadas en el Arco Mediterráneo Peninsular; las cuales, junto a la Comunidad de Madrid, acumulan el 72,3\% del total (Figura 5).

\section{Figura 5 \\ PROCEDENCIA GEOGRÁFICA DE LAS AUTORÍAS}

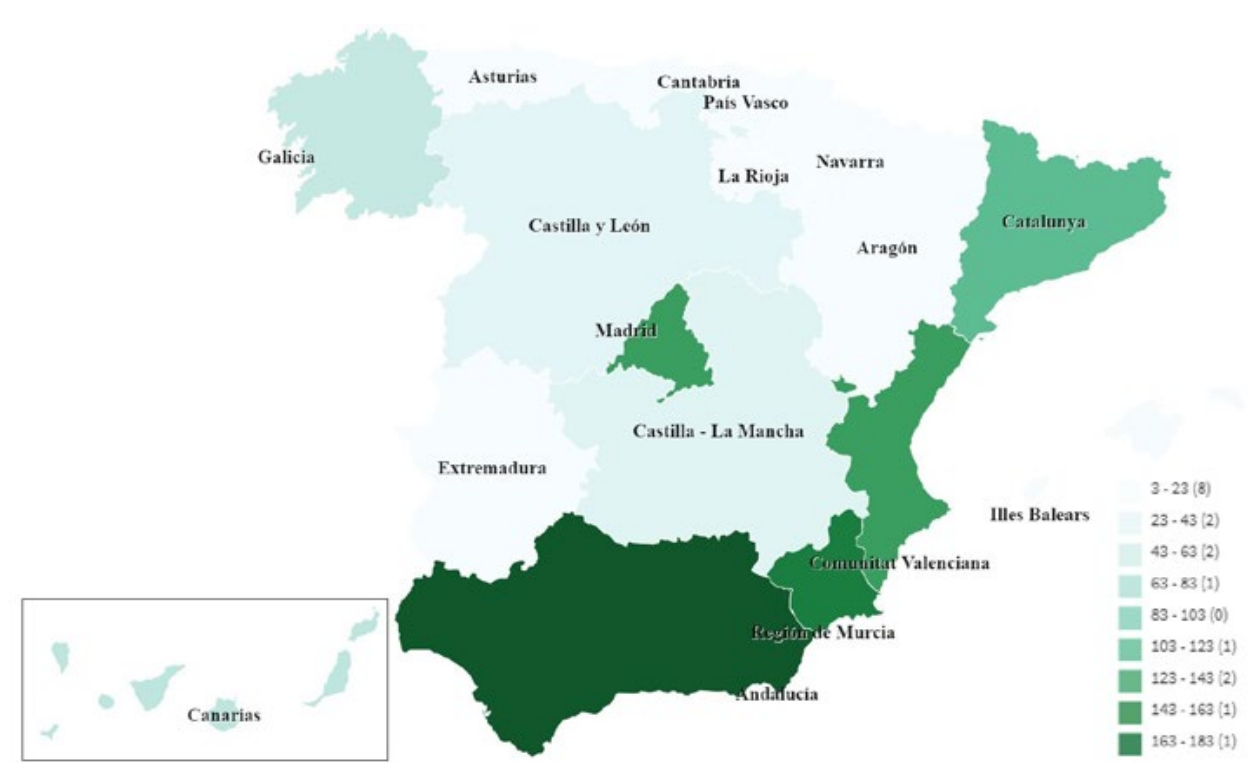

Fuente: Elaboración propia.

\subsection{Temáticas, métodos y territorios}

En 609 artículos se han contabilizado 2.993 palabras clave en total, lo que representa cinco palabras claves por artículo de media (excluyendo nueve artículos sin palabras clave). El número más frecuente de palabras clave por artículo ha sido cinco (30,5\% de los artículos); el $87 \%$ de los artículos han aportado entre tres y seis palabras clave; el 10,8\%, entre siete y diez; y el 1,5\%, más de diez. De las 2.993 palabras clave, 1.758 han sido palabras clave diferentes, debido a la presencia de palabras clave repetidas en varios artículos.

De las 2.993 palabras clave totales, 2.672 fueron temáticas y 321 fueron geográficas o topónimos. Turismo ha sido la palabra clave temática más frecuente: aportada en 147 
artículos de la revista. Las siguientes más utilizadas son turismo cultural (34), turismo rural (28), destino turístico (20), desarrollo local (18), desarrollo sostenible (18), paisaje (18), competitividad (17), patrimonio (17), patrimonio cultural (17), sostenibilidad (17), hoteles (16), política turística (14), oferta turística (13), turismo sostenible (13), desarrollo rural (12), planificación turística (12), demanda turística (11), desarrollo turístico (11) y estacionalidad (11).

\subsubsection{Categorías temáticas de investigación}

En la Figura 6 se presenta la distribución de las 2.672 palabras clave entre las cinco categorías y las 63 subcategorías temáticas (Park et al., 2016). La categoría destino, con el mayor número de subcategorías (25), ha sido la que reunido más palabras clave (1.487; $55,7 \%)$. Las siguientes categorías más frecuentes son: marketing $(441,16,5 \%)$, términos genéricos $(396 ; 14,8 \%)$, demanda $(297 ; 11,1 \%)$ y viaje $(51 ; 1,9 \%)$.

Las 10 subcategorías temáticas más frecuentes han sido (de mayor a menor): atracciones y eventos; segmentación de mercados; turismo; sitio o localización geográfica; teorías y métodos; impactos económicos; instalaciones; política, gobierno y regulación; desarrollo; y gestión de recursos e impactos ambientales. Las 10 subcategorías más frecuentes han concentrado el $51 \%$ de todas las palabras clave (1.362 de 2.672). Las 10 subcategorías temáticas menos frecuentes han sido (de menor a mayor): emociones, previsión de la demanda, tecnología, autenticidad, viaje, experiencias, gestión de las relaciones con los clientes, flujos de viajes (origen-destino), investigación comercial, e infraestructura.

La subcategoría atracciones y eventos, dentro de la categoría destino, ha sido la subcategoría temática con más palabras clave (296). En ella se han englobado términos como paisaje (18), patrimonio (17), patrimonio cultural (17), cultura (9), patrimonio territorial (8), rutas turísticas (6), etc. La nube de palabras de la izquierda de la Figura 7 representa visualmente el contenido de la subcategoría en atracciones y eventos.

La subcategoría segmentación de mercados, dentro de la categoría marketing, ha sido la segunda subcategoría con más palabras clave (177). Se incluyeron aquí términos que expresan tipologías turísticas definidas por el propósito del viaje, como turismo cultural (34), ecoturismo (8), turismo religioso (8), agroturismo (6), enoturismo (6), sol y playa (6), turismo activo (6), turismo residencial (6), etc. La nube de la derecha de la Figura 7 muestra una imagen más completa de las palabras clave incluidas en la subcategoría segmentación de mercados. Debe aclararse que ha sido incluida la subcategoría separada características del mercado, dentro de la categoría demanda, para reunir las palabras claves que aluden a los rasgos demográficos, socioeconómicos, psicográficos y geográficos de los turistas, como turismo accesible, perfil del turista, turismo homosexual, turismo senior, turismo social, etc. 


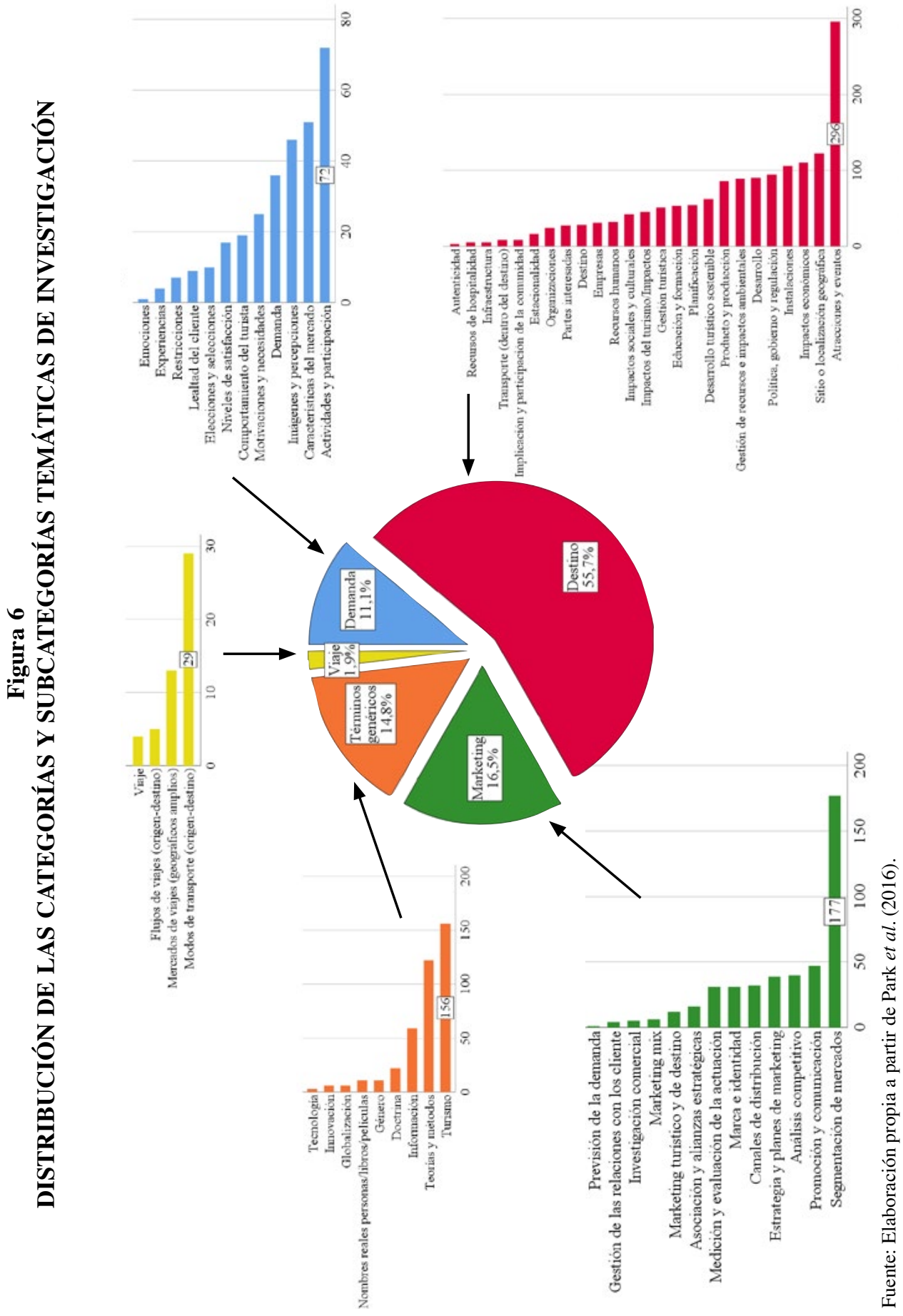




\section{Figura 7 \\ NUBES DE PALABRAS CLAVE DE LAS SUBCATEGORÍAS ATRACCIONES Y EVENTOS Y SEGMENTACIÓN DE MERCADOS}
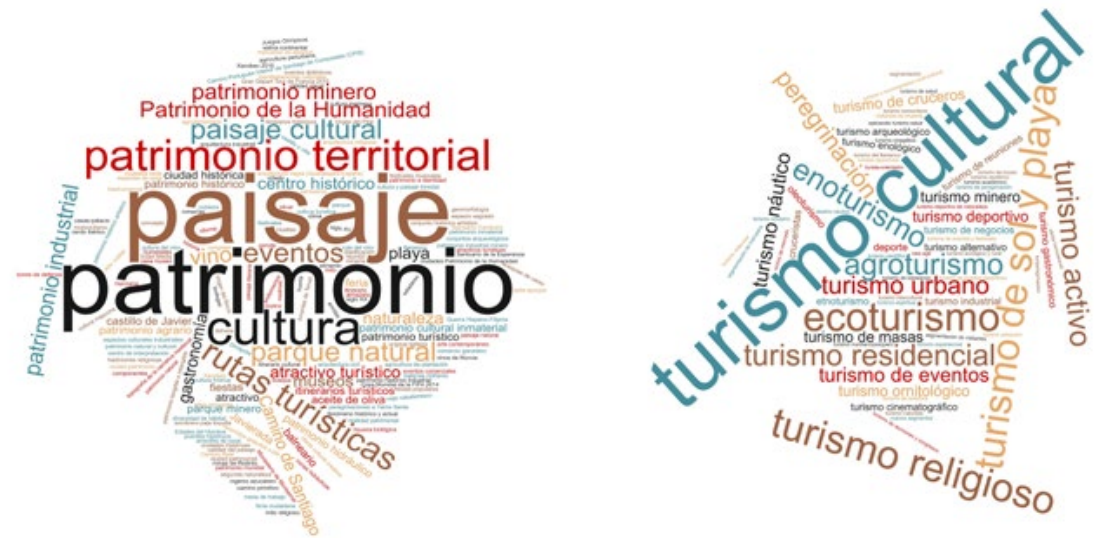

Fuente: Elaboración propia.

La subcategoría teorías y métodos, dentro de la categoría términos genéricos, ha supuesto la quinta categoría con más palabras clave. Mediante palabras clave, muchos autores han aludido a las metodologías, teorías y disciplinas científicas aplicadas en sus investigaciones. De alguna forma, se trata de una subcategoría de naturaleza transversal, que englobó palabras clave como geografía del turismo, sistema de información geográfica, análisis clúster, análisis factorial, análisis de contenido, investigación, metodología, etc. (Figura 8).

\section{Figura 8}

\section{NUBE DE PALABRAS CLAVE DE LA SUBCATEGORÍA TEORÍAS Y MÉTODOS}

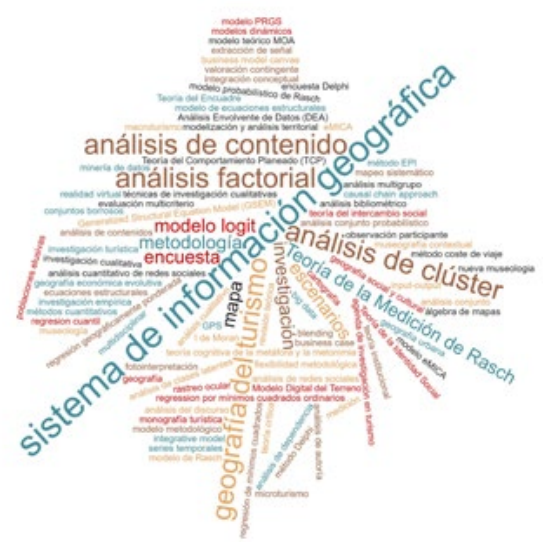

Fuente: Elaboración propia. 


\subsubsection{Análisis geográfico de las palabras clave}

En este apartado se estudia la dimensión geográfica de la investigación publicada en los artículos de Cuadernos de Turismo; por una parte, mediante el análisis de las palabras clave clasificadas en la subcategoría sitio o localización geográfica y, por otra, mediante el análisis y la clasificación de los topónimos mencionados en las palabras clave de la revista.

La subcategoría sitio o localización geográfica, dentro de la categoría destino, ha sido la cuarta más frecuente. En ella se han incluido las palabras clave que aluden a espacios geográficos, como turismo rural, territorio, turismo de interior, turismo de naturaleza, espacios naturales, etc., como se muestra en la nube de palabras de la izquierda de la Figura 9.

Por otra parte, de las 2.996 palabras clave, $321(10,7 \%)$ corresponden a topónimos o nombres propios de lugar. Estas palabras clave se ha analizado por separado de las palabras clave temáticas tratadas en los párrafos anteriores mediante el sistema de clasificación de categorías y subcategorías. Los topónimos más utilizados han sido: España (23), México (14), Andalucía (8), Galicia (8), Murcia (7), Región de Murcia (7), Marruecos (6), Argentina (5), Barcelona (5), Cataluña (5), Islas Canarias (5), Asturias (4), Brasil (3), Islas Baleares (3), Mediterráneo (3) y Navarra (3). El resto de los topónimos se han citado en las palabras clave menos de tres veces. La nube de palabras de la derecha de la Figura 9 contiene todos los topónimos.

\section{Figura 9}

\section{NUBES DE PALABRAS CLAVE DE LA SUBCATEGORÍA SITIO O LOCALIZA- CIÓN GEOGRÁFICA Y DE LOS TOPÓNIMOS}

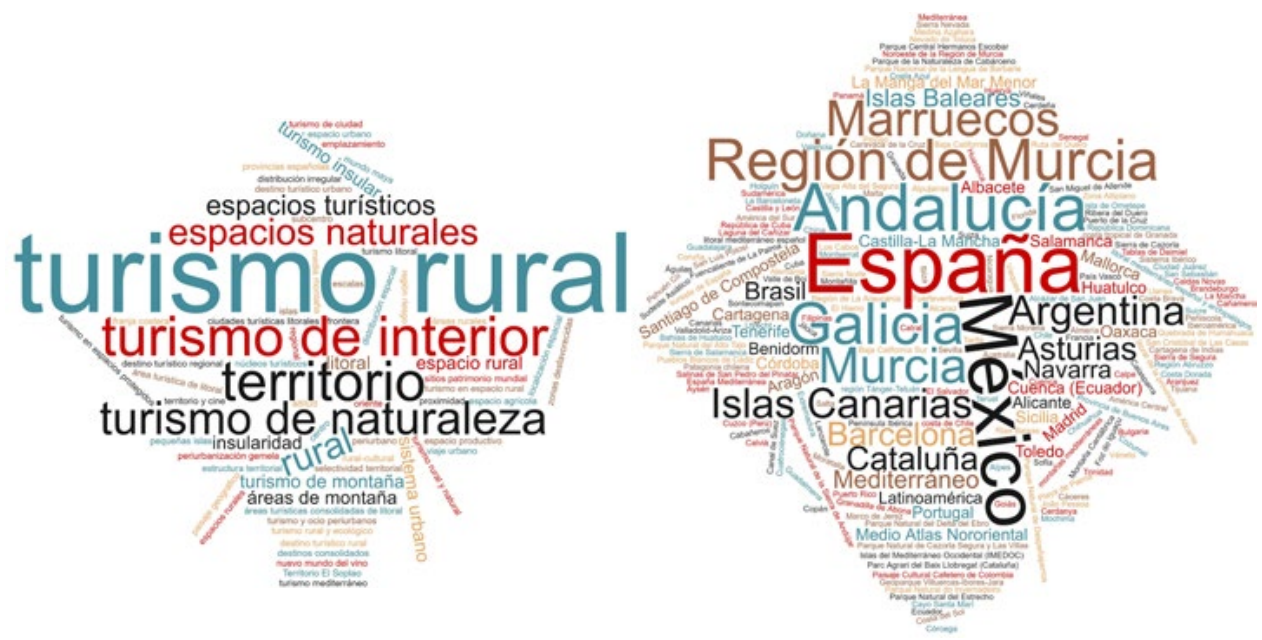

Fuente: Elaboración propia.

Los topónimos se han clasificado según la localización geográfica de los lugares que denominan: los de fuera de España, según continentes y países, y los de España, según comunidades autónomas y provincias. El $61,1 \%$ de los topónimos corresponden a España 
y el 38,9\% son de fuera de nuestro país. Los de fuera de España se han repartido de este modo: América, 68,8\%; Europa, 16,0\%; África, 9,6\%; Asia, 2,4 \%; y Oceanía, 0,8\%. Los países de América con más topónimos han sido: México $(40,7 \%)$, Argentina $(11,6 \%)$, Brasil $(9,3 \%)$, Cuba $(7,0 \%)$, Chile $(5,8 \%)$ y Ecuador $(4,7 \%)$. Los países de Europa con más topónimos han sido Italia y Francia.

Los topónimos de España se han clasificado según el nivel territorial de administración que denominan: municipal $(36,2 \%)$, supramunicipal $(19,4 \%)$, provincial $(5,6 \%)$, supraprovincial $(8,7 \%)$, autonómico $(19,9 \%)$, estatal $(7,1 \%)$ o supraestatal $(3,1 \%)$ (gráfico de la izquierda de la Figura 10). Si consideramos el nivel de los topónimos un indicio de la escala de las áreas geográficas investigadas, se observa que han predominado los estudios de escalas inferiores, mientras que han escaseado los de escalas superiores.

El mapa de la derecha de la Figura 10 muestra que, entre los artículos que han indicado algún topónimo en las palabras clave, han predominado los que han tenido como objeto de investigación territorios de Andalucía (17,6\%), Región de Murcia (14,2\%), Catalunya $(10,8 \%)$, Canarias $(9,1 \%)$, Castilla-La Mancha $(9,1 \%)$, Galicia $(8,5 \%)$ y la Comunitat Valenciana $(6,8 \%)$. Por el contrario, han sido escasos los artículos sobre territorios del País Vasco (1,1\%), Cantabria (1,1\%), Navarra (1,7\%), Comunidad de Madrid (1,7\%), La Rioja (1,7\%), Extremadura (2,3\%), Asturias (2,8\%), Aragón (3,4\%), Islas Baleares $(4,0 \%)$ y Castilla y León $(4,0 \%)$.

Figura 10

\section{NIVEL TERRITORIAL Y MAPA DE LOS TOPÓNIMOS DE ESPAÑA}
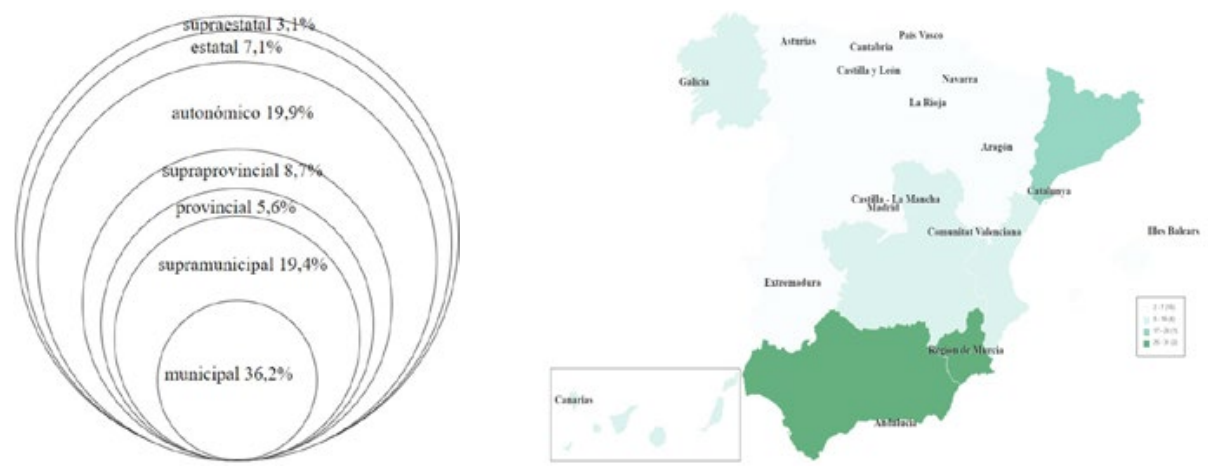

Fuente: Elaboración propia.

Entre los artículos con topónimos que denominan alguno de los niveles territoriales inferiores (municipal, supramunicipal y provincial), han predominado los dedicados a territorios de la Región de Murcia (15\%), provincias de Barcelona (8,3\%), Santa Cruz de Tenerife $(8,3 \%)$, Alicante $(6,7 \%)$, Jaén $(5,8 \%)$, A Coruña $(4,2 \%)$ y Cádiz $(4,2 \%)$, Isla de Mallorca $(4,2 \%)$, y provincias de Albacete $(3,3 \%)$ y Granada $(3,3 \%)$. 


\subsection{Análisis de impacto y citación}

\subsubsection{Impacto científico de la revista: indicador SJR}

En 2018, de acuerdo con la clasificación SCImago Journal \& Country Rank (SJR), basada en datos de Scopus, Cuadernos de Turismo ocupó la posición 87 a de las 102 revistas de la categoría Tourism, Leisure and Hospitality Management (cuarto cuartil). El valor del indicador SJR fue 0,162, el cual expresa el número medio de citas ponderadas recibidas durante un año por los documentos publicados en la revista en los tres años anteriores, y representa una medida de la influencia científica de la revista. El valor del índice h fue 5, el cual expresa el número de artículos (h) que han recibido al menos h citas.

La Figura 11 muestra la evolución en el periodo 2014-2018 del valor del indicador SJR de la revista y de la posición (cuartiles) en las clasificaciones de las tres categorías temáticas. Se observa que la posición de la revista aumentó durante los tres primeros años, pero descendió durante los dos últimos. Por otra parte, comparando la posición de la revista en las tres categorías, aunque la tendencia general fue paralela, siempre estuvo mejor posicionada en Geography, Planning and Development, después en Nature and Landscape Conservation y en tercer lugar en Tourism, Leisure and Hospitality Management.

\section{Figura 11 \\ EVOLUCIÓN DEL SJR DE CUADERNOS DE TURISMO (2014-2018)}

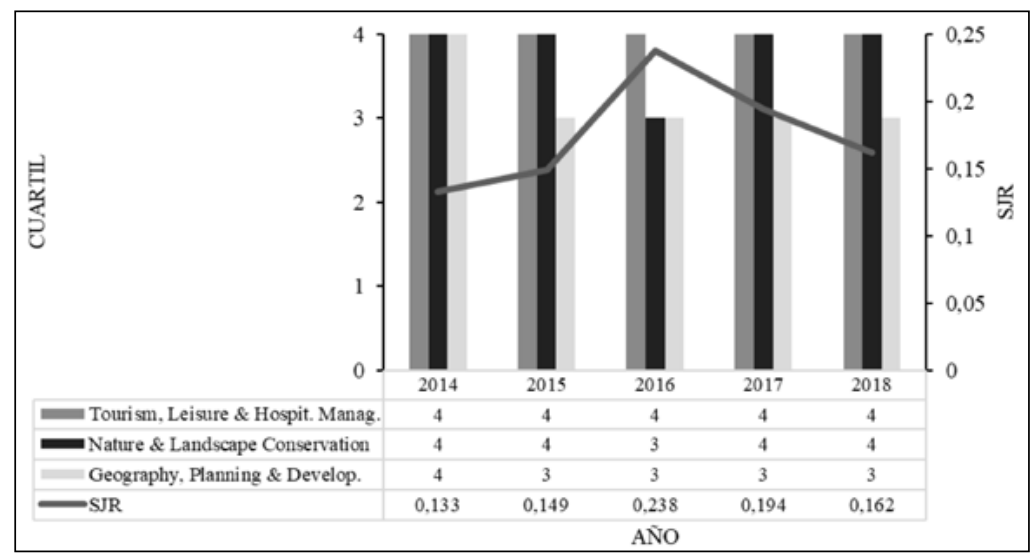

Fuente: Elaboración propia partir de SCImago Journal \& Country Rank.

El gráfico de la izquierda de la Figura 12 muestra la evolución del número total de citas y autocitas recibidas por la revista durante los tres años anteriores. El de la derecha muestra la evolución del número de citas por artículo, totales y externas, recibidas por los artículos publicados durante los tres años previos. Se aprecia un aumento acentuado del número total de citas recibidas y un aumento ligero del número de citas recibidas por artículo. 


\section{Figura 12 \\ EVOLUCIÓN DE LAS CITAS RECIBIDAS EN SCOPUS (2013-2018)}

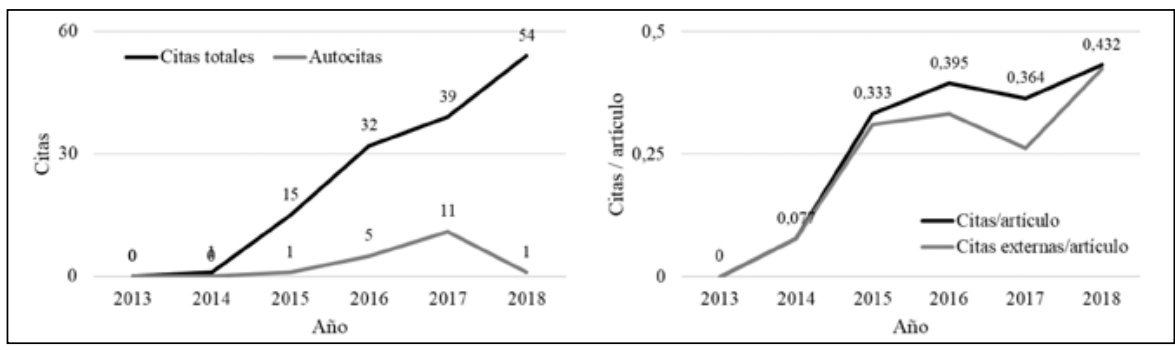

Fuente: Elaboración propia a partir de SCImago Journal \& Country Rank.

\subsubsection{Citas recibidas}

En Scopus constan 261 artículos de Cuadernos de Turismo, publicados en el periodo 2013-2019. A 1 de mayo de 2020, globalmente, los 261 artículos habían recibido 309 citas procedentes de documentos indexados en Scopus, 1,2 citas por artículo de promedio. De los 261 artículos, el 43,7\% contaban con alguna cita, mientras que el 56,3\% no habían recibido cita.

La Tabla 4 muestra los 13 artículos de Cuadernos de Turismo con cinco o más citas (hasta el 1 de mayo de 2020). Además de estos 13 artículos, otros 10 artículos tienen cuatro citas, 17 tres citas, 34 dos citas y 40 una cita. El artículo más citado ha sido "Migración residencial y urbanismo expansivo en el Mediterráneo español", y estudia la "migración internacional de jubilados noreuropeos hacia España y su influencia en el desarrollo del urbanismo expansivo en nuestro país" (Membrado, 2015, p. 259). Publicado en el número 35 de la revista, es obra del profesor del Departamento de Geografía de la Universidad de Valencia Dr. Joan Carles Membrado Tena.

Tabla 4

ARTÍCULOS DE CUADERNOS DE TURISMO MÁS CITADOS EN SCOPUS

\begin{tabular}{lclc}
\hline Título & Año & Autores (Afiliación) & Citas \\
\hline $\begin{array}{l}\text { Migración residencial y urbanismo expansivo } \\
\text { en el Mediterráneo español }\end{array}$ & 2015 & Membrado, Joan C. (UV) & 17 \\
\hline $\begin{array}{l}\text { Recursos turísticos y atractivos turísticos: } \\
\text { conceptualización, clasificación y valoración }\end{array}$ & 2015 & Navarro, Diego (UDA) & 13 \\
\hline $\begin{array}{l}\text { Revisión de la literatura científica sobre } \\
\text { enoturismo en España }\end{array}$ & 2013 & $\begin{array}{l}\text { López-Guzmán, Tomás J. (UCO); } \\
\text { Rdguez., Juan (UCA); Vieira, } \\
\text { Áurea (UCA) }\end{array}$ & 12 \\
\hline
\end{tabular}




\begin{tabular}{lclc}
\hline Título & Año & Autores (Afiliación) & Citas \\
\hline $\begin{array}{l}\text { Adopción del comercio electrónico en el } \\
\text { turismo de nieve y montaña: análisis de la } \\
\text { presencia web de las estaciones de esquí a } \\
\text { través del Modelo eMICA }\end{array}$ & 2016 & $\begin{array}{l}\text { Daries, Natalia (UDL); Cristóbal, } \\
\text { Eduard (UDL); Martín, Eva } \\
\text { (UDL); Mariné, Estela (UDL) }\end{array}$ & 8 \\
\hline $\begin{array}{l}\text { El impacto turístico de los eventos } \\
\text { deportivos: un estudio de caso }\end{array}$ & 2014 & Fernández, María T. (UCA) & 8 \\
\hline $\begin{array}{l}\text { Reflexiones sobre el turismo de proximidad } \\
\text { como una estrategia para el desarrollo local }\end{array}$ & 2013 & $\begin{array}{l}\text { Díaz, Inma (UTM); Llurdés, Joan } \\
\text { C. (UAB) }\end{array}$ & 8 \\
\hline $\begin{array}{l}\text { Chinese tourism in Spain: an analysis of the } \\
\text { tourism product, attractions and itineraries } \\
\text { offered by chinese travel agencies }\end{array}$ & 2016 & Lojo, Aureli (UAB) & 7 \\
\hline $\begin{array}{l}\text { La contribución de una pequeña estación de } \\
\text { esquí al desarrollo de su entorno: el caso de }\end{array}$ & 2014 & $\begin{array}{l}\text { Lasanta, Teodoro (CSIC); Arnáez, } \\
\text { Valdezcaray (La Rioja) }\end{array}$ & 7 \\
\hline $\begin{array}{l}\text { La creación y promoción de experiencias } \\
\text { en un destino turístico. Un análisis de la } \\
\text { investigación y necesidades de actuación }\end{array}$ & 2015 & $\begin{array}{l}\text { Carballo, Rita (ULPGC); Moreno, } \\
\text { Sergio (ULPGC); León, Carmelo }\end{array}$ & 7 \\
\hline $\begin{array}{l}\text { La cuota de mercado como indicador de } \\
\text { competitividad en los destinos turísticos: } \\
\text { sentido y limitaciones }\end{array}$ & 2014 & $\begin{array}{l}\text { Perles, José F. (UA); Ramón, Ana } \\
\text { B. (UA); Sevilla, Martín (UA) }\end{array}$ & 7 \\
\hline $\begin{array}{l}\text { La influencia de las rutas de vuelos de bajo } \\
\text { coste en la elección del destino turístico }\end{array}$ & 2014 & $\begin{array}{l}\text { Saladié, Òscar (URV); Antón, } \\
\text { Salvador (URV); Cortés, Isabel } \\
\text { (IRSTEA); Fdez., Anita (NUBS); } \\
\text { Young, Robert (NUBS) }\end{array}$ & 6 \\
\hline $\begin{array}{l}\text { Discontinuidades y limitaciones de los } \\
\text { últimos planes turísticos de España en } \\
\text { relación a la sostenibilidad ambiental del } \\
\text { turismo de sol y playa }\end{array}$ & 2017 & $\begin{array}{l}\text { Santos, Raquel (URV); Anton, } \\
\text { Salvador (URV); Saladié, Òscar } \\
\text { (URV) }\end{array}$ & 5 \\
\hline $\begin{array}{l}\text { La dimensión turística del patrimonio cultural } \\
\text { de la ciudad de Lorca (Murcia, España) }\end{array}$ & 2015 & Troitiño, Libertad (UCM) & 5 \\
\hline
\end{tabular}

Fuente: Elaboración propia a partir de Scopus.

Las 20 revistas que más veces han citado los artículos de Cuadernos de Turismo indexados en Scopus son: Cuadernos de Turismo (25), Sustainability (16), Boletín de la Asociación de Geógrafos Españoles (14), Espacios (10), Cuadernos Geográficos (8), Documents d'Anàlisi Geogràfica (7), Tourism Economics (5), Anales de Geografía de la Universidad Complutense (4), European Journal of Tourism Research (4), Geojournal of Tourism and Geosites (4), Investigaciones Regionales (4), Tourism Management Perspectives (4), Current Issues in Tourism (3), Estudios Geográficos (3), Intangible Capital (3), Investigaciones Turísticas (3), Journal of Sustainable Tourism (3), Revista de Estudios Regionales (3), Tourism Geographies (3) y Tourism Recreation Research (3). Entre las citas recibidas por los artículos de Cuadernos de Turismo, predominan las de artículos 
publicados en revistas de Geografía, Turismo y Dirección de Empresas; en cierta medida, este dato refleja la dirección disciplinaria de la influencia de la revista.

\subsubsection{Citas emitidas}

Por otra parte, las bibliografías de los 261 artículos de Cuadernos de Turismo indexados en Scopus suman un total de 7.797 referencias. Las 20 revistas más citadas en los artículos publicados en Cuadernos de Turismo indexados en Scopus han sido: Annals of Tourism Research (231), Tourism Management (221), Cuadernos de Turismo (149), Journal of Travel Research (104), Boletín de la Asociación de Geógrafos Españoles (65), International Journal of Hospitality Management (59), Estudios Turísticos (51), Journal of Sustainable Tourism (42), International Journal of Tourism Research (39), International Journal of Contemporary Hospitality Management (37), Journal of Marketing (33), Pasos. Revista de Turismo y Patrimonio Cultural (30), Tourism Economics (29), Journal of Vacation Marketing (28), Journal of Travel and Tourism Marketing (27), Current Issues in Tourism (27), Revista de Análisis Turístico (25), Tourism Geographies (23), Journal of Business Research (23) y Estudios Geográficos (23). De alguna forma, las referencias citadas informan sobre las fuentes de las que se han nutrido las investigaciones publicadas en la revista, y de las áreas disciplinarias que las han influido. Se trata de revistas con gran prestigio, internacionales y españolas, sobre todo de las áreas del Turismo y de la Geografía.

Finalmente, la Tabla 5 muestra los 20 autores más citados en los artículos de Cuadernos de Turismo indexados en Scopus. Como se puede comprobar son autores relevantes, y de amplia trayectoria en el campo de la investigación turística.

\section{Tabla 5 \\ AUTORES MÁS CITADOS EN LOS ARTÍCULOS DE CUADERNOS DE TURISMO}

\begin{tabular}{llll}
\hline Autor & Afiliación & País & Citas \\
\hline C. Michael Hall & University of Canterbury & Nueva Zelanda & 27 \\
\hline Dimitrios Buhalis & Bournemouth University & Reino Unido & 22 \\
\hline Greg Richards & Breda University of Applied Sciences & Países Bajos & 22 \\
\hline Donald Getz & Haskayne School of Business & Canadá & 21 \\
\hline J.R. Brent Ritchie & University of Calgary & Canadá & 20 \\
\hline Ulrike Gretzel & University of Southern California & Estados Unidos & 18 \\
\hline David Harvey & City University of New York & Estados Unidos & 18 \\
\hline José Luis Andrés & Universidad de Murcia & España & 17 \\
\hline Alejandro Mantecón & Universitat d'Alacant & España & 15 \\
\hline Rob Law & Hong Kong Polytechnic University & Hong Kong & 14 \\
\hline Erik Cohen & Hebrew University of Jerusalem & Israel & 13 \\
\hline Daniel Hiernaux & Universidad Autónoma de Querétaro & México & 13 \\
\hline Raquel Huete & Universitat d'Alacant & España & 13 \\
\hline Daniel J. Scott & Universityof Waterloo & Canadá & 13 \\
\hline
\end{tabular}




\begin{tabular}{lllc}
\hline Autor & Afiliación & País & Citas \\
\hline José F. Vera & Universitat d'Alacant & España & 13 \\
\hline Rodolfo Baggio & Università Bocconi & Italia & 12 \\
\hline Xavier Font & University of Surrey & Reino Unido & 12 \\
\hline Bob McKercher & Hong Kong Polytechnic University & Hong Kong & 12 \\
\hline $\begin{array}{l}\text { Juan Ramón Oreja } \\
\text { Rodríguez }\end{array}$ & Universidad de la Laguna & España & 12 \\
\hline $\begin{array}{l}\text { Miguel Ángel } \\
\text { Troitiño }\end{array}$ & Universidad Complutense de Madrid & España & 12 \\
\hline
\end{tabular}

Fuente: Elaboración propia a partir de Scopus.

\section{DISCUSIÓN Y CONCLUSIONES}

Se ha analizado la trayectoria de la revista Cuadernos de Turismo desde varios ángulos: los principales hitos históricos, la distribución de frecuencias de los artículos y las autorías, el origen geográfico e institucional de las autorías, las temáticas y los territorios investigados, el impacto científico, el origen de las citas recibidas y el destino de las emitidas. Durante 22 años, se han publicado 44 números, que suman 609 artículos de 863 autores. A pesar de la presencia de un reducido grupo de autores que han publicado con más frecuencia, el hecho de que más del $90 \%$ de los autores lo sean de uno o dos artículos, pone de manifiesto que la revista ha constituido un canal de publicación atractivo para una multitud de investigadores de diferentes ámbitos científicos. Por otra parte, el dato de que el $81,2 \%$ de las autorías procedan de España pone de manifiesto una concentración en el origen geográfico de los artículos. Sin embargo, a lo largo del tiempo, el origen institucional y el geográfico se han ido extendiendo, desde Murcia a Andalucía, al Arco Mediterráneo Español, al resto de España y a Iberoamérica.

Con respecto a las temáticas tratadas en los artículos de Cuadernos de Turismo, globalmente, están en línea con los resultados obtenidos por Park et al. (2016) sobre 31 revistas internacionales. Este resultado es coherente con el enfoque amplio y multidisciplinar declarado por la revista. En ambos estudios la categoría destino es la predominante, aunque en el caso de Cuadernos de Turismo tiene incluso más presencia; en detrimento de la categoría demanda. Respecto a subcategorías específicas, Cuadernos de Turismo está en sintonía con la investigación turística internacional en la gran atención prestada a temáticas como atracciones y eventos, segmentación de mercados, impactos económicos, instalaciones, desarrollo, gestión de recursos e impactos ambientales, educación y formación, características del mercado y gestión turística. Por contra, algunas temáticas predilectas de Cuadernos de Turismo no destacan relativamente tanto en la investigación turística internacional: sitio o localización geográfica, teorías y métodos, y política, gobierno y regulación. Y viceversa, algunos temas que han despertado gran interés en la investigación internacional no han sido tan relevantes en Cuadernos de Turismo: comportamiento del turista, recursos humanos, empresas, motivaciones y necesidades, servicios y calidad del servicio, canales de distribución y recursos de hospitalidad. Por otro lado, en ambos casos se ha prestado poca atención a temáticas como previsión de la demanda, 
autenticidad, infraestructura, investigación de mercados, flujos turísticos, restricciones y lealtad del cliente. Y algunas carencias temáticas específicas de Cuadernos de Turismo son: emociones, tecnología, gestión de las relaciones con los clientes, experiencias y viaje.

El impacto científico de la revista, comparado con el resto de las revistas presentes en Scopus, es estable, pero relativamente bajo. Por ello, se considera que la revista debería persistir en el esfuerzo por mejorar su posición. Ahora bien, comparado con el resto de las revistas turísticas en español, la influencia es muy alta, por ser la única presente en Scopus hasta 2018, y por ser una de las tres solas indexadas actualmente. Probablemente, la influencia de la revista sea mayor de lo que refleja el índice SJR, sobre todo en el ámbito hispanohablante. Por otra parte, se observa que los artículos más citados suelen ser revisiones, que proporcionan a la comunidad científica útiles marcos de análisis, sobre temas de interés actuales.

Durante las dos últimas décadas se han producido cambios profundos en el entorno de la revista: el turismo ha entrado en la universidad española, con la creación de grados, másteres y doctorados; se ha implantado la evaluación del profesorado, mediante acreditaciones y sexenios; han aumentado continuamente el turismo y la investigación turística, en España y en el mundo; ha nacido y se ha generalizado Internet; ha crecido el acceso abierto; se ha extendido el uso del inglés.

La evolución positiva de la revista demuestra el acierto de su creación, y que la gestión ha sido capaz de adaptarse a las exigencias del entorno. Una muestra de que, tras 22 años, la revista resulta más atractiva que nunca, es el anuncio publicado en el web, a finales de marzo de 2020, ante el aluvión de artículos recibidos: "Envío de nuevos artículos, sólo a partir del próximo mes de diciembre". Entre las causas del "éxito" se hayan el enfoque amplio y multidisciplinar; el cumplimiento de la periodicidad desde el primer número; la edición profesional, gracias al apoyo del Servicio de Publicaciones de la Universidad de Murcia; el esfuerzo para cumplir los criterios de calidad establecidos por el máximo número de bases de datos; la disponibilidad gratuita de sus artículos en Internet desde el comienzo de su edición; la inclusión de un resumen extenso de los artículos en inglés; el equipo de gestores, reducido, estable y experto; y el tejido de una red de confianza entre directores, secretarios, consejeros, autores, revisores, evaluadores, lectores, estudiantes, bibliotecarios...

A la vez, el futuro presenta retos difíciles. Quizás el principal sea la forma de mantener la influencia en el ámbito hispanohablante y, al mismo tiempo, aumentar la internacionalidad, en un entorno académico en el que el inglés ha acontecido la lengua franca. Otros desafíos son la entrada en el JCR, la financiación y el modelo de negocio, el aumento de la competencia, la implantación completa de los metadatos, la visibilidad en nuevos medios digitales de difusión (ResearchGate, Mendeley, Academia...), la presencia en redes sociales (Twitter, blogs...), las puntuaciones en altmétricas y el logro de un alto valor social de la investigación publicada.

\section{AGRADECIMIENTO}

José Antonio Corral agradece al personal del Departamento de Geografía de la Universidad de Murcia, a quien entrevistó, por la información y la hospitalidad ofrecidas durante una estancia de investigación para la elaboración de este artículo en julio de 2019. 


\section{BIBLIOGRAFÍA}

AGÈNCIA DE GESTIÓ D'AJUTS UNIVERSITARIS I DE RECERCA DE LA GENERALITAT DE CATALUNYA (AGAUR) (2014). CARHUS Plus + 2014. Recuperado dehttp://agaur.gencat.cat/es/avaluacio/carhus/carhus-plus-2014/index.html

ALBACH, V.M. y MEDEIROS, M.L. (2020): “Utilização da revisão bibliográfica sistemática em turismo: panorama internacional e ibero-americano dos trabalhos presentes no Scopus e Redalyc", Publicatio-Ciências Sociais Aplicadas, vol. 28, pp. 1-14.

ÁLVAREZ-GARCÍA, J., DURÁN-SÁNCHEZ, A. y DEL RÍO-RAMA, M.C. (2018): "Scientific coverage in community-based tourism: Sustainable tourism and strategy for social development", Sustainability, vol. 10 (4). p. 1158

ANDRÉS, J.L. (1998): "Presentación", Cuadernos de Turismo, nº 1, pp. 7-8.

BENCKENDORFF, P. y YZEHRER, A. (2013): "A network analysis of tourism research", Annals of Tourism Research, vol. 43, pp. 121-149.

CONSELHO DE APERFEIÇOAMENTO DE PESSOAL DO ENSINO SUPERIOR (CAPES) (2016): Plataforma Sucupira. Recuperado de https://sucupira.capes.gov.br/ sucupira/public/consultas/coleta/veiculoPublicacaoQualis/listaConsultaGeralPeriodi$\cos$.jsf

CEBALLOS, C., ARIAS, C., RUIZ, A., SANZ, C. y VÁZQUEZ, I. (2010): "La formación en turismo en España: pasado, presente y futuro en el Nuevo Espacio Europeo de Educación Superior", Cuadernos de Turismo, nº 25, pp. 45-67.

CELDRÁN-BERNABEU, M.A., MAZÓN, J.N., IVARS-BAIDAL, J.A. y VERA-REBOLLO, J.F. (2018): "Smart Tourism. A study on systematic mapping", Cuadernos de Turismo, $\mathrm{n}^{\circ}$ 41, pp. 107-138.

CLARIVATE ANALYTICS (2017): Web of Science ${ }^{T M}$ Core Collection - Emerging Sources Citation Index. Recuperado de http://wokinfo.com/media/pdf/ESCI_Fact_ Sheet.pdf

CORRAL-MARFIL, J.A. (2013): "Estudio bibliométrico de las actas del Congreso de la Asociación Española de Expertos Científicos en Turismo (AECIT, 1994-2012)", Revista de Análisis Turístico, $\mathrm{n}^{\circ}$ 16, pp. 33-44.

CORRAL-MARFIL, J.A. (2014): "La investigación turística en España”, en García, A. (Coord.) 20 años de la actividad turística en España. Madrid, Síntesis, pp. 321-336.

CORRAL-MARFIL, J.A. y CÀNOVES, G. (2016): "An Approach to Tourism Research in Spain”, en Munar, A.M. y Jamal, T. (Eds.) Tourism Research Paradigms: Critical and Emergent Knowledges. Bingley, Reino Unido, Emerald, pp. 167-189.

CORRAL-MARFIL, J.A., RODRÍGUEZ, I.M., VARGAS, Á. y CÀNOVES, G. (2015): "Estudio de la investigación turística a través de las coautorías de artículos: cálculo de indicadores de colaboración y análisis de redes sociales. El caso de las universidades catalanas", Pasos. Revista de Turismo y Patrimonio Cultural, vol. 13 (4), pp. 789-803.

CORRAL-MARFIL, J.A. y ESPEJO MARÍN, C. (2020): "Hitos, autorías, temáticas, territorios e impacto de la revista Cuadernos de Turismo", comunicación presentada al XIV Congreso Internacional de la Academia Mexicana de Investigación Turística. Mazatlán, Universidad Autónoma de Occidente. 
CUADERNOS DE TURISMO (1998): "Normas para la publicación de originales", Cuadernos de Turismo, $\mathrm{n}^{\circ} 1$.

CUADERNOS DE TURISMO (2001): "Normas para la publicación de originales”, Cuadernos de Turismo, $\mathrm{n}^{\circ} 7$.

CUADERNOS DE TURISMO (2009): "Normas para la publicación de originales”, Cuadernos de Turismo, $\mathrm{n}^{\circ} 23$.

CUADERNOS DE TURISMO (2010): "Normas para la publicación de originales”, Cuadernos de Turismo, $\mathrm{n}^{\circ} 26$.

DELGADO, E. y MARTÍN-MARTÍN, A. (2019): Índice $H$ de las revistas científicas españolas según Google ScholarMetrics (2014-2018). https://doi.org/10.13140/ RG.2.2.36649.13923

DÍAZ-CARRIÓN, I.A. y VIZCAINO-SUÁREZ, P. (2019): “Tourism and gender research in Brazil and Mexico", Tourism, Culture and Communication, vol. 19 (4), pp. 277-289.

DURÁN-SÁNCHEZ, A., ÁLVAREZ-GARCÍA, J., DEL RÍO-RAMA, M.C. y ROSADOCEBRIÁN, B. (2019): "Science mapping of the knowledge base on tourism innovation", Sustainability, vol. 11 (12), p. 3352.

DURÁN-SÁNCHEZ, A., DEL RÍO-RAMA, M.C. y ÁLVAREZ-GARCÍA, J. (2017): "Bibliometric analysis of publications on wine tourism in the databases Scopus and WoS", European Research on Management and Business Economics, vol. 23 (1), pp. 8-15.

EC3M RICS (2020). CIRC: Clasificación Integrada de Revistas Científicas. Recuperado dehttps://clasificacioncirc.es

ESPEJO MARÍN, C. y GARCÍA MARÍN, R. (2020): Cuadernos de Turismo en 2019. Recuperado de: https://revistas.um.es/turismo/reports

ESPEJO MARÍN, C. (2011): "Presentación”, Cuadernos de Turismo, n 27, pp. 9-11.

ESPEJO MARÍN, C. (2012): "Cuadernos de Turismo", en Fundación Española para la Ciencia y la Tecnología (FECYT) (Ed.) Manual de buenas prácticas en edición de revistas científicas. Madrid, FECYT, pp. 55-62.

ESPEJO MARÍN, C. y GARCÍA MARÍN, R. (2020): Cuadernos de Turismo en 2019. Murcia, Departamento de Geografía de la Universidad de Murcia, 16 de enero de 2020. Recuperado dehttps://revistas.um.es/turismo/reports

FUNDACIÓN ESPAÑOLA PARA LA CIENCIA Y LA TECNOLOGÍA. FECYT (2018): Guía de evaluación de la sexta convocatoria de evaluación de la calidad editorial y científica de las revistas científicas españolas. Recuperado de https://evaluacionarce. fecyt.es/Publico/Bases/__Recursos/2018GuiaEval6Conv_FECYT.pdf

FUNDACIÓN ESPAÑOLA PARA LA CIENCIA Y LA TECNOLOGÍA. FECYT (2020): Ranking de visibilidad e impacto de revistas científicas españolas de humanidades y ciencias sociales con sello de calidad FECYT. Recuperado de https://calidadrevistas. fecyt.es/sites/default/files/informes/2020_02_ranking_revistas_sello_fecyt.pdf

GOOGLE SCHOLAR METRICS. Recuperado de https://scholar.google.com/intl/en/scholar/metrics.html\#metrics

GURSOY, D. y SANDSTROM, J.K. (2016): “An Updated Ranking of Hospitality and Tourism Journals", Journal of Hospitality and Tourism Research, vol. 40 (1), pp. 3-18. 
GÜZELLER, C.O. y ÇELIKER, N. (2019): "Bibliometrical analysis of Asia Pacific Journal of Tourism Research", Asia Pacific Journal of Tourism Research, vol. 24 (1), pp. 108-120.

KOSEOGLU, M.A.,RAHIMI, R.,OKUMUS, F. y LIU, J. (2016): "Bibliometric studies in tourism", Annals of Tourism Research, vol. 61, pp. 180-198.

LÓPEZ-BONILLA, J.M., GRANADOS-PEREA, C. y LÓPEZ-BONILLA, L.M. (2017): "Primera generación de autores con difusión internacional en la investigación turística española", Revista Española de Documentación Científica, vol. 40 (3), pp. 1-18.

LÓPEZ-BONILLA, J.M., GRANADOS-PEREA, C. y LÓPEZ-BONILLA, L.M. (2018a): “Autores prolíficos líderes en la investigación turística española”, Transinformação, vol. 30 (1), pp. 39-50.

LÓPEZ-BONILLA, J.M., GRANADOS-PEREA, C. y LÓPEZ-BONILLA, L.M. (2018b): "Producción científica española en turismo: Un análisis de autoría basado en revistas internacionales con alto impacto y visibilidad”, Cuadernos de Turismo, $\mathrm{n}^{\circ} 41$, pp. 343-367.

MAULEÓN-MÉNDEZ, E., GENOVART-BALAGUER, J., MERIGÓ, J.M. y MULETFORTEZA, C. (2018): "Sustainable tourism research towards twenty-five years of the Journal of Sustainable Tourism", Advances in Hospitality and Tourism Research, vol. 6 (1), pp. 23-46.

MCKERCHER, B. y TUNG, V. (2015): "Publishing in tourism and hospitality journals: Is the past a prelude to the future?", Tourism Management, vol. 50, pp. 306-315.

MCKERCHER, B. y TUNG, V. (2016):'The rise of fractional authors", Annals of Tourism Research, vol. 61, pp. 213-267.

MEMBRADO, J.C. (2015): "Migración residencial y urbanismo expansivo en el Mediterráneo español", Cuadernos de Turismo, no 35, pp. 259-286.

MENEGUEL, C.R.A., PALAU, S. y MUNDET, L. (2019): “Análisis bibliométrico de la investigación turística sobre la ciudad de Girona”, REVISTA ROSA DOS VENTOSTurismo e Hospitalidade, vol. 11 (3), pp. 598-614.

MERIGÓ, J.M., MULET-FORTEZA, C., VALENCIA, C. y LEW, A.A. (2019): “Twenty years of Tourism Geographies: a bibliometric overview", Tourism Geographies, vol. 21 (5), pp. 881-910.

MILL, R.C. y MORRISON, A.M. (2006): Tourism System (5 ed.). Dubuque, EE.UU., Kendall Hunt.

MOKHTARI, H., SOLTANI-NEJAD, N., MIREZATI, S.Z. y SABERI, M.K. (2020): “A bibliometric and altmetric analysis of Anatolia: 1997-2018”, Anatolia, pp. 1-17.

MULET-FORTEZA, C., MARTORELL-CUNILL, O., MERIGÓ, J.M., GENOVARTBALAGUER, J. y MAULEÓN-MÉNDEZ, E. (2018): “Twenty five years of the Journal of Travel \& Tourism Marketing: a bibliometric ranking”, Journal of Travel and Tourism Marketing, vol. 35 (9), pp. 1.201-1.221.

ORGANIZACIÓN MUNDIAL DEL TURISMO (2019): Panorama del turismo internacional, edición 2019. Madrid, OMT. https://doi.org/10.18111/9789284421237

OSORIO, M. (2016): "Revista Estudios y Perspectivas en Turismo. Calidad científica y editorial, temáticas e indicadores bibliométricos", Estudios y Perspectivas en Turismo, vol. 25 (4), pp. 539-557. 
PARK, J., WU, B., MORRISON, A.M., SHEN, Y.Y.,CONG, L. y LI, M. (2016): The Tourism System Research Categorization Framework", Asia Pacific Journal of Tourism Research, vol. 21 (9), pp. 968-1.000.

PEÑA, A.R., JIMÉNEZ, M., RUIZ, J. y PONTÓN, T. (2019): "La producción científica en el "Sector de Hostelería y Turismo": Un análisis bibliométrico de las tesis doctorales españolas en el periodo 1978-2018”, Investigaciones Turísticas, n 18, pp. 71-94.

PINASSI, A. y YERCOLANI, P. (2015): “Geografía del turismo: análisis de las publicaciones científicas en revistas turísticas. El caso de Argentina”, Cuadernos de Geografía: Revista Colombiana de Geografía, vol. 24 (1), pp. 214-230.

PORIA, Y. y TIMOTHY, D.J. (2014): “Where are the children in tourism research?", Annals of Tourism Research, vol. 47, pp. 93-95.

QIAN, J., LAW, R., WEI, J. y WU, Y. (2019): “Trends in Global Tourism Studies: A Content Analysis of the Publications in Tourism Management", Journal of Quality Assurance in Hospitality and Tourism, vol. 20 (6), pp. 753-768.

RAMÓN-CARDONA, J. (2017): "Reflexiones sobre el turismo en el ámbito académico español", Redmarka. Revista de Marketing Aplicado, vol. 18, pp. 83-115.

RAMÓN-CARDONA, J. y SÁNCHEZ-FERNÁNDEZ, M.D. (2019): "Principales focos de investigación en turismo y hospitalidad de los países iberoamericanos", Espacios, vol. 40 (4), pp. 1-21.

RODRÍGUEZ-LÓPEZ, M.E., ALCÁNTARA-PILAR, J.M., DEL BARRIO-GARCÍA, S. y MUÑOZ-LEIVA, F. (2020): "A review of restaurant research in the last two decades: A bibliometric analysis", International Journal of Hospitality Management, vol. 87, p. 102387.

SEVERT, D.E.,TESONE, D.V., BOTTORFF, T.J. y CARPENTER, M.L. (2009): “A world ranking of the top 100 hospitality and tourism programs", Journal of Hospitality and Tourism Research, vol. 33 (4), pp. 451-470.

TOKIĆ, K. y TOKIĆ, I. (2015): "Bibliometric Analysis from the Perspective of a Croatian Tourism Journal", Qualitative and Quantitative Methods in Libraries, vol. 4 (4), pp. 927-936.

UNIVERSIDAD DE BARCELONA (2020): MIAR 2020 live. Matriz de Información para el Análisis de Revistas [en línea]. Recuperado de http://miar.ub.edu

WEB OF SCIENCE MASTER JOURNAL LIST (2020): Cuadernos de Turismo. Recuperado de https://mjl.clarivate.com:/search-results?issn=1139-7861\&hide_ exact_match_fl=true\&utm_source $=$ mjl\&utm_medium $=$ share-by-link \&utm_ campaign=search-results-share-this-journal

XIAO, H. y SMITH, S.L.J. (2006): “Case studies in tourism research: A state-of-the-art analysis", Tourism Management, vol. 27 (5), pp. 738-749.

ŽUPIČ, I. y ČATER, T. (2015): "Bibliometric Methods in Management and Organization", Organizational Research Methods, vol. 18 (3), pp. 429-472. 


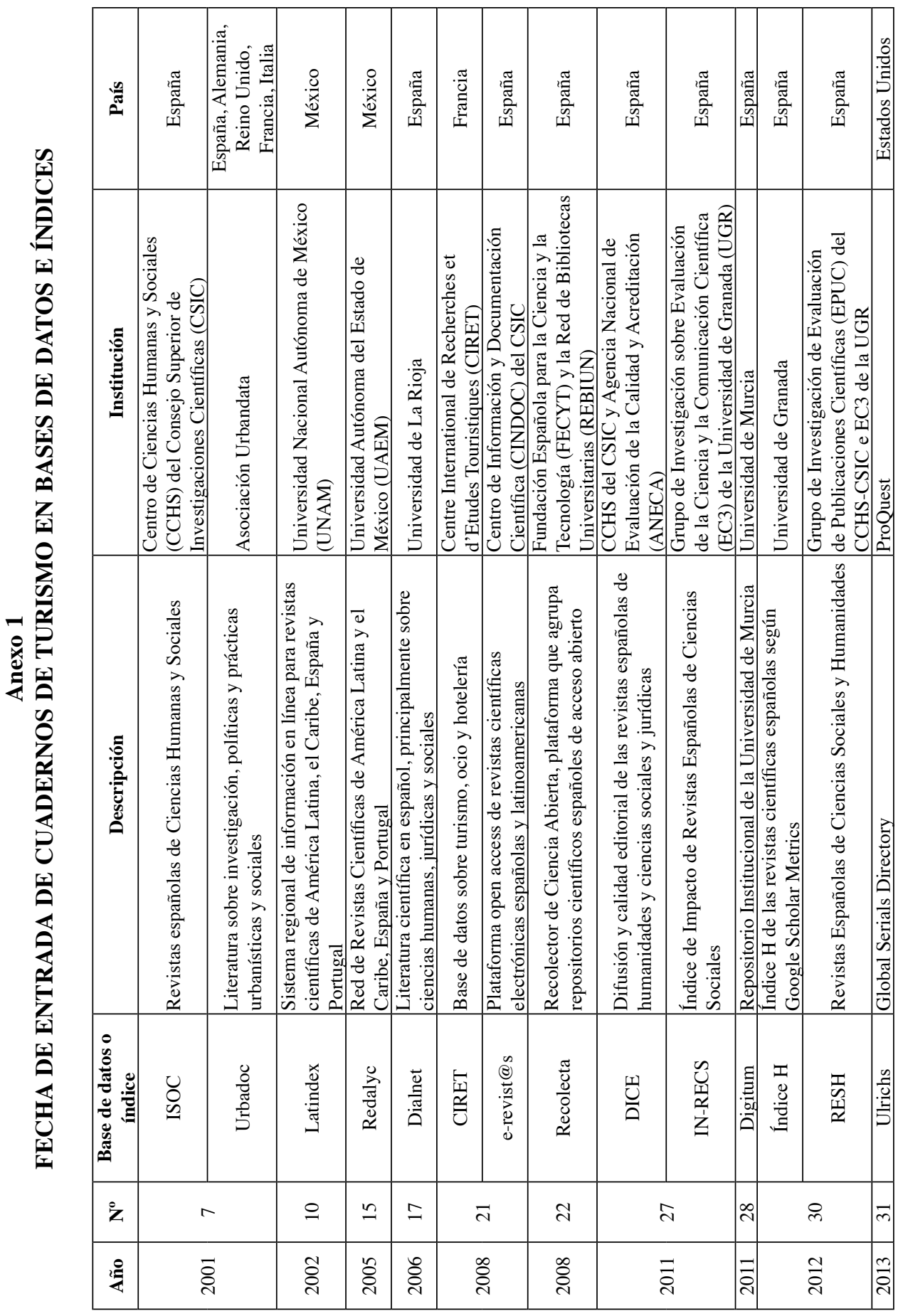




\begin{tabular}{|c|c|c|c|c|c|c|c|c|c|c|c|c|c|}
\hline 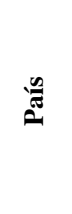 & 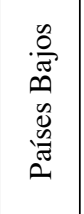 & 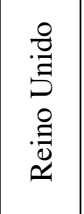 & 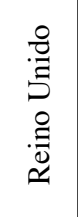 & 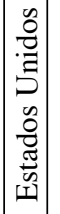 & 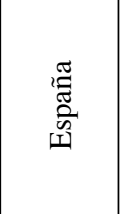 & 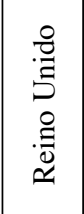 & 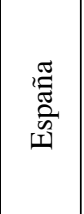 & 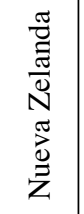 & 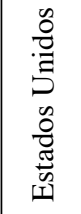 & 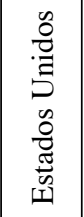 & 鱼 & $\begin{array}{l}\underset{0}{0} \\
\stackrel{0}{Z} \\
D_{0} \\
Z\end{array}$ & 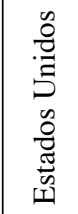 \\
\hline & $\begin{array}{l}\frac{\bar{d}}{\Delta} \\
\frac{0}{\Delta} \\
I\end{array}$ & 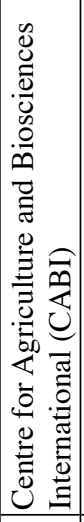 & 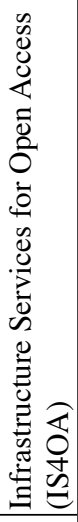 & 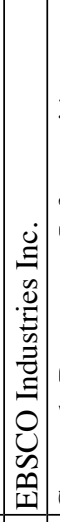 & 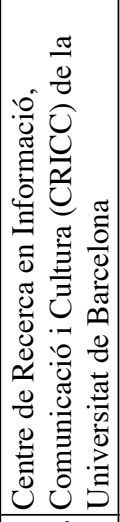 & 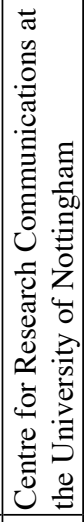 & 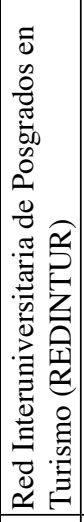 & 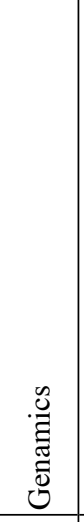 & 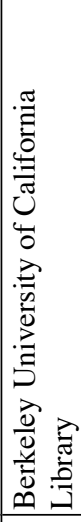 & 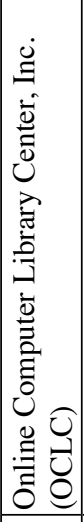 & 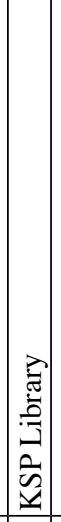 & 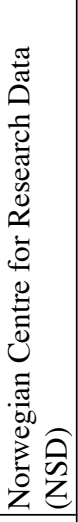 & 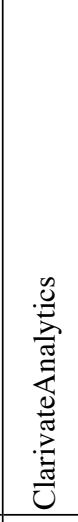 \\
\hline & 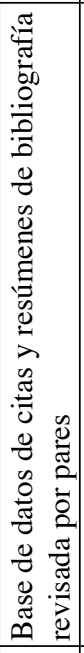 & 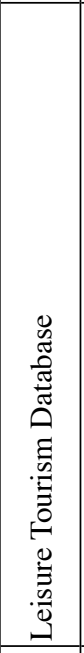 & 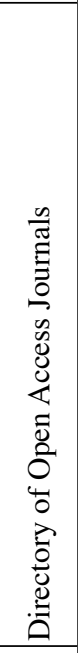 & 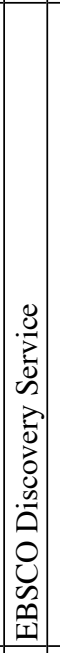 & 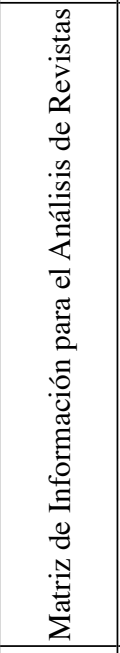 & 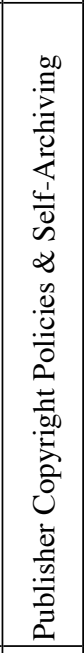 & 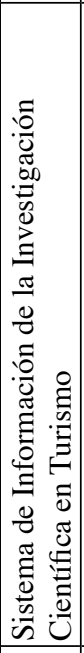 & 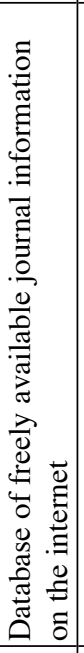 & 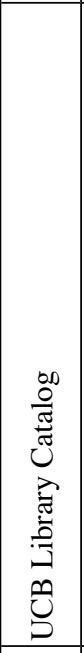 & 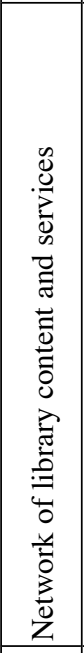 & 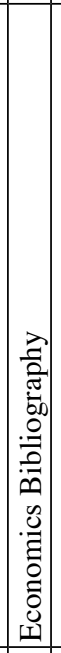 & 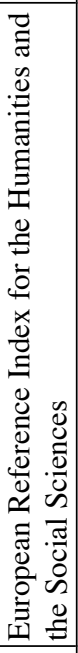 & 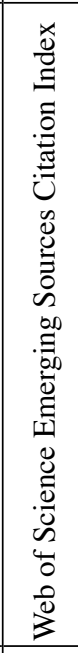 \\
\hline 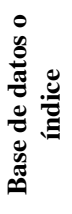 & 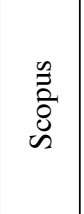 & 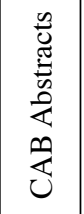 & 灾 & $\left|\begin{array}{l}0 \\
0 \\
\tilde{D} \\
0 \\
1\end{array}\right|$ & $\frac{a}{d}$ & 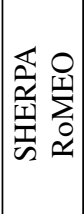 & 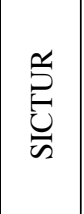 & 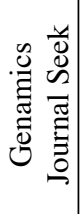 & 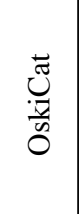 & $\frac{\pi}{0}$ & 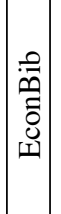 & 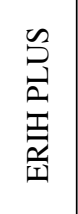 & 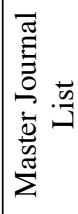 \\
\hline Z & \multicolumn{7}{|c|}{$m$} & \multicolumn{3}{|c|}{$\ddot{n}$} & $\begin{array}{l}0 \\
\varnothing\end{array}$ & \multicolumn{2}{|c|}{$m$} \\
\hline 㝵 & \multicolumn{7}{|c|}{$\stackrel{+}{\stackrel{N}{*}}$} & \multicolumn{3}{|c|}{$\frac{n}{2}$} & $\frac{n}{\tilde{c}}$ & \multicolumn{2}{|c|}{$\bar{N}$} \\
\hline
\end{tabular}




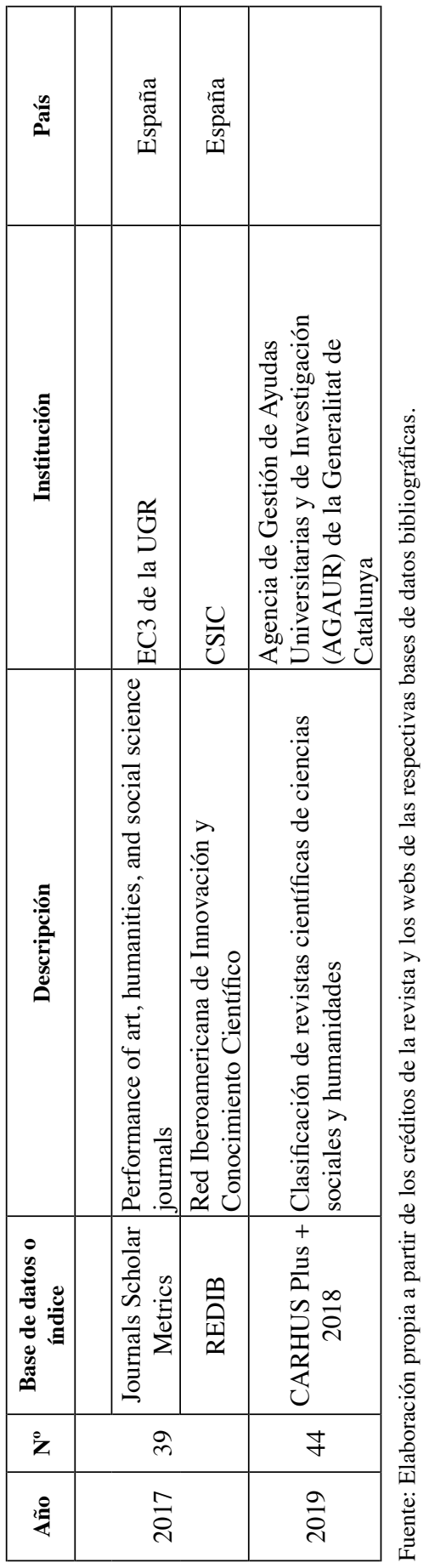


